MATHEMATICS OF COMPUTATION

Volume 80, Number 273, January 2011, Pages 297-325
S 0025-5718(2010)02378-6

Article electronically published on July 14, 2010

\title{
HOW TO INTEGRATE A POLYNOMIAL OVER A SIMPLEX
}

\author{
VELLEDA BALDONI, NICOLE BERLINE, JESUS A. DE LOERA, MATTHIAS KÖPPE, \\ AND MICHÈLE VERGNE
}

\begin{abstract}
This paper starts by settling the computational complexity of the problem of integrating a polynomial function $f$ over a rational simplex. We prove that the problem is NP-hard for arbitrary polynomials via a generalization of a theorem of Motzkin and Straus. On the other hand, if the polynomial depends only on a fixed number of variables, while its degree and the dimension of the simplex are allowed to vary, we prove that integration can be done in polynomial time. As a consequence, for polynomials of fixed total degree, there is a polynomial time algorithm as well. We explore our algorithms with some experiments. We conclude the article with extensions to other polytopes and discussion of other available methods.
\end{abstract}

\section{INTRODUCTION}

Let $\Delta$ be a $d$-dimensional rational simplex inside $\mathbb{R}^{n}$ and let $f \in \mathbb{Q}\left[x_{1}, \ldots, x_{n}\right]$ be a polynomial with rational coefficients. We consider the problem of how to efficiently compute the exact value of the integral of the polynomial $f$ over $\Delta$, which we denote by $\int_{\Delta} f \mathrm{~d} m$. Here we use the integral Lebesgue measure $\mathrm{d} m$ on the affine hull $\langle\Delta\rangle$ of the simplex $\Delta$, defined below in Section 2.1. This normalization of the measure occurs naturally in Euler-Maclaurin formulas for a polytope $P$, which relate sums over the lattice points of $P$ with certain integrals over the various faces of $P$. For this measure, the volume of the simplex and every integral of a polynomial function with rational coefficients are rational numbers. Thus the result has a representation in the usual (Turing) model of computation. This is in contrast to other normalizations, such as the induced Euclidean measure, where irrational numbers appear.

The main goals of this article are to discuss the computational complexity of the problem and to provide methods to do the computation that are both theoretically efficient and have reasonable performance in concrete examples.

Computation of integrals of polynomials over polytopes is fundamental for many applications. We already mentioned summation over lattice points of a polytope. They also make an appearance in recent results in optimization problems connected to moment matrices 28. In geometric modeling, the generation of smooth multivariate polynomial splines is equivalent to integrals over slices of polyhedra (see Chapter 4 of [32]). Integrals over polyhedra are also commonly computed in finite element methods, where the domain is decomposed into cells (typically simplices)

Received by the editor February 16, 2009 and, in revised form, September 25, 2009.

2010 Mathematics Subject Classification. Primary 68W30; Secondary 52B55. 
via a mesh, and complicated functions are approximated by polynomials (see for instance [38]). When studying a random univariate polynomial $p(x)$ whose coefficients are independent random variables in certain intervals, the probability distribution for the number of real zeros of $p(x)$ is given as an integral over a polytope 9 . Integrals over polytopes also play a very important role in statistics; see, for instance, [30. We remark that among all polytopes, simplices are the fundamental case to consider for integration, since any convex polytope can be triangulated into finitely many simplices.

Regarding the computational complexity of our problem, one can ask what happens with integration over arbitrary polytopes. It is very educational to look first at the case when $f$ is the constant polynomial 1 , and the answer is simply a volume. It has been proved already that computing the volume of polytopes of varying dimension is \#P-hard [19, 12, 25, 29] and that even approximating the volume is hard [20]. More recently, in [34] it was proved that computing the centroid of a polytope is \#P-hard. In contrast, for a simplex, the volume is given by a determinant, which can be computed in polynomial time. One of the key contributions of this paper is to settle the computational complexity of integrating a non-constant polynomial over a simplex. Before we can state our results let us better understand the input and output of our computations. Our output will always be the rational number $\int_{\Delta} f \mathrm{~d} m$ in the usual binary encoding. The $d$-dimensional input simplex will be represented by its vertices $\mathbf{s}_{1}, \ldots, \mathbf{s}_{d+1}$ (a $V$-representation), but note that, in the case of a simplex, one can go from its representation as a system of linear inequalities (an $H$-representation) to a $V$-representation in polynomial time, simply by computing the inverse of a matrix.

Thus the encoding size of $\Delta$ is given by the number of vertices, the dimension, and the largest binary encoding size of the coordinates among vertices. Computations with polynomials also require that one specifies concrete data structures for reading the input polynomial and to carry on the calculations. There are several possible choices. One common representation of a polynomial is as a sum of monomial terms with rational coefficients. Some authors assume the representation is dense (polynomials are given by a list of the coefficients of all monomials up to a given total degree $r$ ), while other authors assume it is sparse (polynomials are specified by a list of exponent vectors of monomials with non-zero coefficients, together with their coefficients). Another popular representation is by straight-line programs. A straight-line program which encodes a polynomial is, roughly speaking, a program without branches which enables us to evaluate it at any given point (see [15, 31] and the references therein). As we explain in Section 2, general straight-line programs are too compact for our purposes, so instead we restrict ourselves to a subclass we call single-intermediate-use (division-free) straight-line programs, or SIU straightline programs for short. The precise definition and explanation will appear in Section 2 but for now the reader should think that polynomials are represented as fully parenthesized arithmetic expressions involving binary operators + and $\times$.

Now we are ready to state our first result.

Theorem 1 (Integrating general polynomials over a simplex is hard). The following problem is NP-hard.

Input:

$\left(\mathrm{I}_{1}\right)$ numbers $d, n \in \mathbb{N}$ in unary encoding,

$\left(\mathrm{I}_{2}\right)$ affinely independent rational vectors $\mathbf{s}_{1}, \ldots, \mathbf{s}_{d+1} \in \mathbb{Q}^{n}$ in binary encoding, 
$\left(\mathrm{I}_{3}\right)$ an SIU straight-line program $\Phi$ encoding a polynomial $f \in \mathbb{Q}\left[x_{1}, \ldots, x_{n}\right]$ with rational coefficients.

Output, in binary encoding:

$\left(\mathrm{O}_{1}\right)$ the rational number $\int_{\Delta} f \mathrm{~d} m$, where $\Delta \subseteq \mathbb{R}^{n}$ is the simplex with vertices $\mathbf{s}_{1}, \ldots, \mathbf{s}_{d+1}$ and $\mathrm{d} m$ is the integral Lebesgue measure of the rational affine subspace $\langle\Delta\rangle$.

But we can also prove the following positive results.

Theorem 2 (Efficient integration of polynomials of a fixed effective number of variables). For every fixed number $D \in \mathbb{N}$, there exists a polynomial-time algorithm for the following problem.

Input:

$\left(\mathrm{I}_{1}\right)$ numbers $d, n, M \in \mathbb{N}$ in unary encoding,

$\left(\mathrm{I}_{2}\right)$ affinely independent rational vectors $\mathbf{s}_{1}, \ldots, \mathbf{s}_{d+1} \in \mathbb{Q}^{n}$ in binary encoding,

$\left(\mathrm{I}_{3}\right)$ a polynomial $f \in \mathbb{Q}\left[X_{1}, \ldots, X_{D}\right]$ represented by either an SIU straight-line program $\Phi$ of formal degree at most $M$ or a sparse or dense monomial representation of total degree at most $M$,

$\left(\mathrm{I}_{4}\right)$ a rational matrix $L$ with $D$ rows and $n$ columns in binary encoding, the rows of which define $D$ linear forms $\mathbf{x} \mapsto\left\langle\ell_{j}, \mathbf{x}\right\rangle$ on $\mathbb{R}^{n}$.

Output, in binary encoding:

$\left(\mathrm{O}_{1}\right)$ the rational number $\int_{\Delta} f\left(\left\langle\ell_{1}, \mathbf{x}\right\rangle, \ldots,\left\langle\ell_{D}, \mathbf{x}\right\rangle\right) \mathrm{d} m$, where $\Delta \subseteq \mathbb{R}^{n}$ is the simplex with vertices $\mathbf{s}_{1}, \ldots, \mathbf{s}_{d+1}$ and $\mathrm{d} m$ is the integral Lebesgue measure of the rational affine subspace $\langle\Delta\rangle$.

In particular, the computation of the integral of a power of one linear form can be done by a polynomial time algorithm. This becomes false already if one considers powers of a quadratic form instead of powers of a linear form. Actually, we prove Theorem 1 by looking at powers $Q^{M}$ of the Motzkin-Straus quadratic form of a graph.

Our method relies on properties of integrals of exponentials of linear forms. A. Barvinok had previously investigated these integrals and their computational complexity (see [3], [5]).

As we will see later, when its degree is fixed, a polynomial has a polynomial size representation in either the SIU straight-line program encoding or the sparse or dense monomial representation, and one can switch between the three representations efficiently. The notion of a formal degree of an SIU straight-line program will be defined in Section 2 ,

Corollary 3 (Efficient integration of polynomials of fixed degree). For every fixed number $M \in \mathbb{N}$, there exists a polynomial-time algorithm for the following problem. Input:

$\left(\mathrm{I}_{1}\right)$ numbers $d, n \in \mathbb{N}$ in unary encoding,

$\left(\mathrm{I}_{2}\right)$ affinely independent rational vectors $\mathbf{s}_{1}, \ldots, \mathbf{s}_{d+1} \in \mathbb{Q}^{n}$ in binary encoding,

$\left(\mathrm{I}_{3}\right)$ a polynomial $f \in \mathbb{Q}\left[x_{1}, \ldots, x_{n}\right]$ represented by either an SIU straight-line program $\Phi$ of formal degree at most $M$ or a sparse or dense monomial representation of total degree at most $M$.

Output, in binary encoding: 
$\left(\mathrm{O}_{1}\right)$ the rational number $\int_{\Delta} f(\mathbf{x}) \mathrm{d} m$, where $\Delta \subseteq \mathbb{R}^{n}$ is the simplex with vertices $\mathbf{s}_{1}, \ldots, \mathbf{s}_{d+1}$ and $\mathrm{d} m$ is the integral Lebesgue measure of the rational affine subspace $\langle\Delta\rangle$.

Actually, we give two interesting methods that prove Corollary 3 , First, we simply observe that a monomial with total degree $M$ involves at most $M$ variables. The other method is related to the polynomial Waring problem: we decompose a homogeneous polynomial of total degree $M$ into a sum of $M$-th powers of linear forms.

In [27. Lasserre and Avrachenkov compute the integral $\int_{\Delta} f(\mathbf{x}) \mathrm{d} m$ when $f$ is a homogeneous polynomial, in terms of the corresponding polarized symmetric multilinear form (Proposition 18). We show that their formula also leads to a proof of Corollary 3. Furthermore, several other methods can be used for integration of polynomials of fixed degree. We discuss them in Section 4 .

This paper is organized as follows: After some preparation in Section 2, the main theorems are proved in Section 3. In Section 4, we discuss extensions to other convex polytopes and give a survey of the complexity of other algorithms. Finally, in Section 5, we describe the implementation of the two methods of Section 3, and we report on a few computational experiments.

\section{Preliminaries}

In this section we prepare for the proofs of the main results.

2.1. Integral Lebesgue measure on a rational affine subspace of $\mathbb{R}^{n}$. On $\mathbb{R}^{n}$ itself we consider the standard Lebesgue measure, which gives volume 1 to the fundamental domain of the lattice $\mathbb{Z}^{n}$. Now, if we integrate using this measure over a full-dimensional polyhedron, we will obtain a rational number. Nevertheless, if the polyhedron is not full-dimensional, say it lies in a proper subspace $L$, this may yield irrational numbers. To avoid problems in our model of computation, we adjust the measure slightly.

Let $L$ be a rational linear subspace of dimension $d \leq n$. The lattice points inside $L$ form an Abelian group of rank $d$. This group acts on $L$ via translation, the images of a single point under the group action form an orbit of the action. A fundamental domain is a subset of the space which contains exactly one point from each of these orbits. We normalize the Lebesgue measure on $L$ in such a way that the volume of the fundamental domain of the intersected lattice $L \cap \mathbb{Z}^{n}$ is 1 . Then for any affine subspace $L+$ a parallel to $L$, we define the integral Lebesgue measure $\mathrm{d} m$ by translation. For example, the diagonal of the unit square has length 1 instead of $\sqrt{2}$. Note that the standard Lebesgue measure and the integral measure coincide when the lattice is full-dimensional, and the usual Lebesgue volume will be a scalar multiple of the rational number we compute here.

2.2. Encoding polynomials for integration. We now explain our encoding of polynomials as SIU straight-line programs and justify our use of this encoding. We say that a polynomial $f$ is represented as a (division-free) straight-line program $\Phi$ if there is a finite sequence of polynomial functions of $\mathbb{Q}\left[x_{1}, \ldots, x_{n}\right]$, namely $q_{1}, \ldots, q_{k}$, the so-called intermediate results, such that each $q_{i}$ is either a variable $x_{1}, \ldots, x_{n}$, an element of $\mathbb{Q}$, or either the sum or the product of two preceding polynomials in the sequence and such that $q_{k}=f$. A straight-line program allows us to describe in polynomial space many polynomials which otherwise would need to be 
TABLE 1. The representation of $\left(x_{1}^{2}+\cdots+x_{n}^{2}\right)^{k}$ as a straight-line program

\begin{tabular}{|c|c|}
\hline Intermediate & Comment \\
\hline $\begin{aligned} q_{1} & =0 \\
q_{2} & =x_{1} \\
q_{3} & =q_{2} \cdot q_{2} \\
q_{4} & =q_{1}+q_{3} \\
q_{5} & =x_{2} \\
q_{6} & =q_{5} \cdot q_{5} \\
q_{7} & =q_{4}+q_{6}\end{aligned}$ & $\begin{array}{l}\text { Thus } q_{4}=x_{1}^{2} . \\
\text { Now } q_{7}=x_{1}^{2}+x_{2}^{2} \text {. }\end{array}$ \\
\hline $\begin{aligned} q_{3 n-1} & =x_{n} \\
q_{3 n} & =q_{3 n-1} \cdot q_{3 n-1} \\
q_{3 n+1} & =q_{3 n-2}+q_{3 n} \\
q_{3 n+2} & =1 \\
q_{3 n+3} & =q_{3 n+2} \cdot q_{3 n+1} \\
q_{3 n+4} & =q_{3 n+3} \cdot q_{3 n+1}\end{aligned}$ & Now $q_{3 n+1}=x_{1}^{2}+\cdots+x_{n}^{2}$ \\
\hline$q_{3 n+k+2}=q_{3 n+k+1} \cdot q_{3 n+1}$ & Final result. \\
\hline
\end{tabular}

described with exponentially many monomial terms. For example, think of the representation of $\left(x_{1}^{2}+\cdots+x_{n}^{2}\right)^{k}$ as monomials versus its description with only $3 n+k+2$ intermediate results; see Table 1. The number of intermediate results of a straight-line program is called its length. To keep track of constants we define the size of an intermediate result as one, unless the intermediate result is a constant, in which case its size is the binary encoding size of the rational number. The size of a straight-line program is the sum of the sizes of the intermediate results. The formal degree of an intermediate result $q_{i}$ is defined recursively in the obvious way, namely as 0 if $q_{i}$ is a constant of $\mathbb{Q}$, as 1 if $q_{i}$ is a variable $x_{j}$, as the maximum of the formal degrees of the summands if $q_{i}$ is a sum, and as the sum of the formal degrees of the factors if $q_{i}$ is a product. The formal degree of the straight-line program $\Phi$ is the formal degree of the final result $q_{k}$. Clearly the total degree of a polynomial is bounded by the formal degree of any straight-line program which represents it.

A favorite example to illustrate the benefits of a straight-line program encoding is that of the symbolic determinant of an $n \times n$ matrix. Its dense representation as monomials has size $\Theta(n !)$, but it can be computed in $\mathrm{O}\left(n^{3}\right)$ operations by Gaussian elimination. See the book [15] as a reference for this concept.

From a monomial representation of a polynomial of degree $M$ and $n$, variables it is easy to encode it as a straight-line program: first, by going in increasing degree we can write a straight-line program that generates all monomials of degree at most $M$ in $n$ variables. Then for each of them compute the product of the monomial with its coefficient; thus the length doubles. Finally, successively add each term. This gives a final length bounded above by four times the number of monomials of degree at most $M$ in $n$ variables.

Straight-line programs are quite natural in the context of integration. One would certainly not expand $\left(x_{1}^{2}+\cdots+x_{n}^{2}\right)^{k}$ to carry on numeric integration when we can 
TABLE 2. The representation of a straight-line program for $x^{2^{k}}$, using iterated squaring

\begin{tabular}{cl}
\hline Intermediate & Comment \\
\hline$q_{1}=x$ & \\
$q_{2}=q_{1} \cdot q_{1}$ & \\
$q_{3}=q_{2} \cdot q_{2}$ & \\
$\vdots$ & \\
$q_{k+1}=q_{k} \cdot q_{k}$ & Final result. \\
\hline
\end{tabular}

easily evaluate it as a function. More importantly, straight-line programs are suitable as an input and output encoding and data structure in certain symbolic algorithms for computations with polynomials, like factoring; see [15. Since straightline programs can be very compact, the algorithms can handle polynomials whose input and output encodings have an exponential size in a sparse monomial representation.

However, a problem with straight-line programs is that this input encoding can be so compact that the output of many computational questions cannot be written efficiently in the usual binary encoding. For example, while one can encode the polynomial $x^{2^{k}}$ with a straight-line program with only $k+1$ intermediate results (see Table 2), when we compute the value of $x^{2^{k}}$ for $x=2$, or the integral $\int_{0}^{2} x^{2^{k}} \mathrm{~d} x=$ $2^{2^{k}+1} /\left(2^{k}+1\right)$, the binary encoding of the output has a size of $\Theta\left(2^{k}\right)$. Thus the output, given in binary encoding, turns out to be exponentially larger than the input encoding. We remark that the same difficulty arises if we choose a sparse input encoding of the polynomial, where not only the coefficients but also the exponent vectors are encoded in binary encoding (rather than the usual unary encoding for the exponent vectors).

This motivates the following variation of the notion of straight-line program: We say a (division-free) straight-line program is single-intermediate-use, or SIU for short, if every intermediate result is used only once in the definition of other intermediate results. (However, the variables $x_{1}, \ldots, x_{n}$ can be used arbitrarily often in the definition of intermediate results.) With this definition, all ways to encode the polynomial $x^{2^{k}}$ require at least $2^{k}$ multiplications. An example SIU straight-line program is shown in Table 3. Clearly, single-intermediate-use straightline programs are equivalent, in terms of expressiveness and encoding complexity, to fully parenthesized arithmetic expressions using binary operators + and $\times$.

2.3. Efficient computation of a truncated product of an arbitrary number of polynomials in a fixed number of variables. The following result will be used in several situations.

Lemma 4. For every fixed number $D \in \mathbb{N}$, there exists a polynomial time algorithm for the following problem.

Input: a number $M$ in unary encoding, a sequence of $k$ polynomials $P_{j} \in$ $\mathbb{Q}\left[X_{1}, \ldots, X_{D}\right]$ of total degree at most $M$, in dense monomial representation.

Output: the product $P_{1} \cdots P_{k}$ truncated at degree $M$. 
TABLE 3. The representation of a single-intermediate-use straightline program for $x^{2^{k}}$; note that the iterated squaring method cannot be used

\begin{tabular}{|c|c|}
\hline Intermediate & Comment \\
\hline $\begin{array}{l}q_{1}=x \\
q_{2}=x \\
q_{3}=q_{1} \cdot q_{2}\end{array}$ & $\begin{array}{l}\text { Now } q_{3}=x^{2} \text {, and } q_{1} \text { and } q_{2} \text { can- } \\
\text { not be used anymore. }\end{array}$ \\
\hline $\begin{array}{l}q_{4}=x \\
q_{5}=q_{3} \cdot q_{4} \\
\quad \vdots\end{array}$ & Thus $q_{5}=x^{3}$. \\
\hline $\begin{array}{l}q_{2^{k+1}-2}=x \\
q_{2^{k+1}-1}=q_{2^{k+1}-3} \cdot q_{2^{k+1}-2}\end{array}$ & Final result. \\
\hline
\end{tabular}

Proof. We start with the product of the first two polynomials. We compute the monomials of degree at most $M$ in this product. This takes $\mathrm{O}\left(M^{2 D}\right)$ elementary rational operations, and the maximum encoding length of any coefficient in the product is also polynomial in the input data length. Then we multiply this truncated product with the next polynomial, truncating at degree $M$, and so on. The total computation takes $\mathrm{O}\left(k M^{2 D}\right)$ elementary rational operations.

\section{Proofs of the Main Results}

Our aim is to perform an efficient computation of $\int_{\Delta} f \mathrm{~d} m$ where $\Delta$ is a simplex and $f$ a polynomial. We will first prove that this is not possible for $f$ of varying degree under the assumption that $\mathrm{P} \neq \mathrm{NP}$. More precisely, we prove that, under this assumption, an efficient computation of $\int_{\Delta} Q^{M} \mathrm{~d} m$ is not possible, where $Q$ is a quadratic form and $M$ is allowed to vary.

In the next subsection we present an algorithm to efficiently compute the integral $\int_{\Delta} f \mathrm{~d} m$ in some particular situations, most notably the case of arbitrary powers of linear forms.

3.1. Hardness for polynomials of non-fixed degree. For the proof of Theorem 11 we need to extend the following well-known result of Motzkin and Straus [33. In this section, we denote by $\Delta$ the $(n-1)$-dimensional canonical simplex $\left\{\mathrm{x} \in \mathbb{R}^{n}\right.$ : $\left.x_{i} \geq 0, \sum_{i=1}^{n} x_{i}=1\right\}$, and we again denote by $\mathrm{d} m$ the integral Lebesgue measure on the hyperplane $\left\{\mathbf{x} \in \mathbb{R}^{n}: \sum_{i=1}^{n} x_{i}=1\right\}$.

Just for this subsection, in order to simplify our calculations, we normalized further so that $\Delta$ has volume 1 instead of $1 / d$ ! (this is just a multiple factor difference). For a function $f$ on $\Delta$, denote as usual $\|f\|_{\infty}=\max _{\mathbf{x} \in \Delta}|f(\mathbf{x})|$ and $\|f\|_{p}=\left(\int_{\Delta}|f|^{p} \mathrm{~d} m\right)^{1 / p}$, for $p \geq 1$. Recall that the clique number of a graph $G$ is the largest number of vertices of a complete subgraph of $G$.

Theorem 5 (Motzkin-Straus). Let $G$ be a graph with $n$ vertices, edge set $E(G)$ and clique number $\omega(G)$. Let $Q_{G}(\mathbf{x})$ be the Motzkin-Straus quadratic form $\frac{1}{2} \sum_{(i, j) \in E(G)} x_{i} x_{j}$. Then $\left\|Q_{G}(\mathbf{x})\right\|_{\infty}=\frac{1}{2}\left(1-\frac{1}{\omega(G)}\right)$. 
Our first result might be of independent interest, as it shows that integrals of polynomials over simplices can carry very interesting combinatorial information. This result builds on the theorem of Motzkin and Straus, using the proof of the well-known relation $\|f\|_{\infty}=\lim _{p \rightarrow \infty}\|f\|_{p}$.

Lemma 6. Let $G$ be a graph with $n$ vertices and clique number $\omega(G)$. Let $Q_{G}(\mathbf{x})$ be the Motzkin-Straus quadratic form. Then for $p \geq 4(e-1) n^{3} \ln \left(32 n^{2}\right)$, the clique number $\omega(G)$ is equal to $\left\lceil\frac{1}{1-2\left\|Q_{G}\right\|_{p}}\right\rceil$.

To prove Lemma 6 we will first prove the following intermediate result.

Lemma 7. For $\varepsilon>0$ we have

$$
\left(\left\|Q_{G}\right\|_{\infty}-\varepsilon\right)\left(\frac{\varepsilon}{4}\right)^{(n-1) / p} \leq\left\|Q_{G}\right\|_{p} \leq\left\|Q_{G}\right\|_{\infty} .
$$

Proof. The right-hand side inequality follows from the normalization of the measure, as $\left|Q_{G}(\mathbf{x})\right| \leq\left\|Q_{G}\right\|_{\infty}$, for all $\mathbf{x} \in \Delta$.

In order to obtain the other inequality, we use Hölder's inequality $\int_{\Delta}|f g| \mathrm{d} m \leq$ $\|f\|_{p}\|g\|_{q}$, where $q$ is such that $\frac{1}{p}+\frac{1}{q}=1$. For any (say) continuous function $f$ on $\Delta$, let us denote by $\Delta(f, \varepsilon)$ the set $\left\{\mathbf{x} \in \Delta:|f(\mathbf{x})| \geq\|f\|_{\infty}-\varepsilon\right\}$ and take for $g$ the characteristic function of $\Delta(f, \varepsilon)$. We obtain

$$
\left(\|f\|_{\infty}-\varepsilon\right)(\operatorname{vol} \Delta(f, \varepsilon))^{1 / p} \leq\|f\|_{p} .
$$

Let a be a point of $\Delta$ where the maximum of $Q_{G}$ is attained. Since $\frac{\partial Q_{G}}{\partial x_{i}}=$ $\sum_{(i, j) \in E(G)} x_{j}$ we know that $0 \leq \frac{\partial Q_{G}}{\partial x_{i}} \leq 1$ for $\mathbf{x} \in \Delta$. Since $\Delta$ is convex, we conclude that for any $\mathbf{x} \in \Delta$,

$$
0 \leq Q_{G}(\mathbf{a})-Q_{G}(\mathbf{x}) \leq \sum_{i=1}^{n}\left|a_{i}-x_{i}\right|
$$

Thus $\Delta\left(Q_{G}, \varepsilon\right)$ contains the set $C_{\varepsilon}=\left\{\mathbf{x} \in \Delta: \sum_{i=1}^{n}\left|a_{i}-x_{i}\right|<\varepsilon\right\}$. We claim that $\operatorname{vol}\left(C_{\varepsilon}\right) \geq\left(\frac{\varepsilon}{4}\right)^{n-1}$. This claim proves the left inequality of the lemma when we apply it to (1).

Consider the dilated simplex $\frac{\varepsilon / 2}{1+\varepsilon / 2} \Delta$ and the translated set $P_{\varepsilon}=\frac{\mathbf{a}}{1+\varepsilon / 2}+\frac{\varepsilon / 2}{1+\varepsilon / 2} \Delta$. Clearly $P_{\varepsilon}$ is contained in $\Delta$. Moreover, for $\mathbf{x} \in P_{\varepsilon}$, we have $\sum_{i=1}^{n}\left|a_{i}-x_{i}\right| \leq$ $\frac{\varepsilon}{1+\varepsilon / 2} \leq \varepsilon$, hence $P_{\varepsilon}$ is contained in $C_{\varepsilon}$. Since $\operatorname{vol}(\Delta)=1$ for the normalized measure, the volume of $P_{\varepsilon}$ is equal to $\left(\frac{\varepsilon / 2}{1+\varepsilon / 2}\right)^{n-1}$. Hence $\operatorname{vol}\left(P_{\varepsilon}\right) \geq(\varepsilon / 4)^{n-1}$. This finishes the proof.

Proof of Lemma 6. In the inequalities of Lemma 7, we substitute the relation $\left\|Q_{G}\right\|_{\infty}=\frac{1}{2}\left(1-\frac{1}{\omega(G)}\right)$, given by Motzkin-Straus's theorem (Theorem 5). We obtain

$$
\left(\frac{1}{2}\left(1-\frac{1}{\omega(G)}\right)-\varepsilon\right)(\varepsilon / 4)^{\frac{n-1}{p}} \leq\left\|Q_{G}\right\|_{p} \leq \frac{1}{2}\left(1-\frac{1}{\omega(G)}\right) .
$$

Let us rewrite these inequalities as

$$
\frac{1}{1-2\left\|Q_{G}\right\|_{p}} \leq \omega(G) \leq \frac{1}{1-\frac{2\left\|Q_{G}\right\|_{p}}{(\varepsilon / 4)^{(n-1) / p}}-2 \varepsilon} .
$$


We only need to prove that for $\varepsilon=\frac{1}{8 n^{2}}$ and $p \geq 4(e-1) n^{3} \ln \left(32 n^{2}\right)$ we have

$$
0 \leq L(p):=\frac{1}{1-\frac{2\left\|Q_{G}\right\|_{p}}{(\varepsilon / 4)^{\frac{n-1}{p}}}-2 \varepsilon}-\frac{1}{1-2\left\|Q_{G}\right\|_{p}}<1 .
$$

Let us write

$$
\delta_{p}=\left\|Q_{G}\right\|_{p}\left(\frac{1}{(\varepsilon / 4)^{\frac{n-1}{p}}}-1\right)=\left\|Q_{G}\right\|_{p}\left(\left(32 n^{2}\right)^{\frac{n-1}{p}}-1\right) .
$$

Thus $L(p)$ in (3) now becomes

$$
L(p)=\frac{1}{1-2\left\|Q_{G}\right\|_{p}}\left(\frac{1}{1-2 \frac{\delta_{p}+\varepsilon}{1-2\left\|Q_{G}\right\|_{p}}}-1\right) .
$$

Since $\left\|Q_{G}\right\|_{p} \leq \frac{1}{2}\left(1-\frac{1}{\omega(G)}\right) \leq \frac{1}{2}$, we have a bound for $\delta_{p}$ :

$$
0 \leq \delta_{p} \leq \frac{1}{2}\left(\left(32 n^{2}\right)^{\frac{n-1}{p}}-1\right) .
$$

Let $A=\left(\frac{4}{\varepsilon}\right)^{n-1}=\left(32 n^{2}\right)^{n-1}$. Since we assumed $p \geq 4(e-1) n^{3} \ln \left(32 n^{2}\right)$, we have $0 \leq \frac{\ln A}{p}<1$; hence $0 \leq A^{1 / p}-1<(e-1) \frac{\ln A}{p}$. We obtain

$$
0 \leq \delta_{p} \leq \frac{e-1}{2} \frac{(n-1) \log \left(32 n^{2}\right)}{p} \leq \frac{1}{8 n^{2}} .
$$

Since $\omega(G) \leq n$, we have $1-2\left\|Q_{G}\right\|_{p} \geq 1 / n$. Hence we have

$$
\frac{2\left(\delta_{p}+\varepsilon\right)}{1-2\left\|Q_{G}\right\|_{p}} \leq \frac{1}{2 n} \leq \frac{1}{2}
$$

Finally, for any number $0<\alpha<1 / 2$ we have $\frac{1}{1-\alpha}<1+2 \alpha$. Hence applying this fact to (4), with $\alpha=2 \frac{\delta_{p}+\varepsilon}{1-2\left\|Q_{G}\right\|_{p}}$ we get

$$
L(p)<\frac{1}{1-2\left\|Q_{G}\right\|_{p}}\left(\frac{4(\delta+\varepsilon)}{1-2\left\|Q_{G}\right\|_{p}}\right) \leq 4 n^{2}\left(\delta_{p}+\varepsilon\right) \leq 1 .
$$

This proves (3) and the lemma.

Proof of Theorem 1. The problem of deciding whether the clique number $\omega(G)$ of a graph $G$ is greater than a given number $K$ is a well-known NP-complete problem 21. From Lemma 6 we see that checking this is the same as checking that for $p=4(e-1) n^{3} \ln \left(32 n^{2}\right)$ the integral part of $\int_{\Delta}\left(Q_{G}\right)^{p} \mathrm{~d} m$ is less than $K^{p}$. Note that the polynomial $Q_{G}(\mathbf{x})^{p}$ is a power of a quadratic form and can be encoded as a SIU straight-line program of length $\mathrm{O}\left(n^{3} \log n \cdot|E(G)|\right)$. If the computation of the integral $\int_{\Delta} f \mathrm{~d} m$ of a polynomial $f$ could be done in polynomial time in the input size of $f$, we could then verify the desired inequality in polynomial time as well.

3.2. An extension of a formula of Brion. In this section, we obtain several expressions for the integrals $\int_{\Delta} e^{\ell} \mathrm{d} m$ and $\int_{\Delta} \ell_{1}^{M_{1}} \cdots \ell_{D}^{M_{D}} \mathrm{~d} m$, where $\Delta \subset \mathbb{R}^{n}$ is a simplex, $\ell, \ell_{1}, \ldots, \ell_{D}$ are linear forms on $\mathbb{R}^{n}$ and $\mathrm{d} m$ again denotes the integral Lebesgue measure. The first formula, (5) in Lemma 8 , is obtained by elementary iterated integration on the standard simplex. It leads to a computation of the integral $\int_{\Delta} \ell_{1}^{M_{1}} \cdots \ell_{D}^{M_{D}} \mathrm{~d} m$ in terms of the Taylor expansion of a certain analytic function associated to $\Delta$ (Corollary 11), hence to a proof of the complexity result of Theorem 2, 
In the case of one linear form $\ell$ which is regular, we recover in this way the "short formula" of Brion as Corollary 12. This result was first obtained by Brion as a particular case of his theorem on polyhedra [13].

Lemma 8. Let $\Delta$ be the simplex that is the convex hull of $(d+1)$ affinely independent vertices $\mathbf{s}_{1}, \mathbf{s}_{2}, \ldots, \mathbf{s}_{d+1}$ in $\mathbb{R}^{n}$, and let $\ell$ be an arbitrary linear form on $\mathbb{R}^{n}$. Then

$$
\int_{\Delta} e^{\ell} \mathrm{d} m=d ! \operatorname{vol}(\Delta, \mathrm{d} m) \sum_{\mathbf{k} \in \mathbb{N}^{d+1}} \frac{\left\langle\ell, \mathbf{s}_{1}\right\rangle^{k_{1}} \cdots\left\langle\ell, \mathbf{s}_{d+1}\right\rangle^{k_{d+1}}}{(|\mathbf{k}|+d) !}
$$

where $|\mathbf{k}|=\sum_{j=1}^{d+1} k_{j}$.

Proof. Using an affine change of variables, it is enough to prove (5) when $\Delta$ is the $d$-dimensional standard simplex $\Delta_{s t} \subset \mathbb{R}^{d}$ defined by

$$
\Delta_{s t}=\left\{\mathrm{x} \in \mathbb{R}^{d}: x_{i} \geq 0, \sum_{i=1}^{d} x_{i} \leq 1\right\} \text {. }
$$

The volume of $\Delta_{s t}$ is equal to $\frac{1}{d !}$. In the case of $\Delta_{s t}$, the vertex $\mathbf{s}_{j}$ is the basis vector $\mathbf{e}_{j}$ for $1 \leq j \leq d$ and $s_{d+1}=0$. Let $\langle\ell, x\rangle=\sum_{j=1}^{d} a_{j} x_{j}$. Then (5) becomes

$$
\int_{\Delta_{s t}} e^{a_{1} x_{1}+\cdots+a_{d} x_{d}} \mathrm{~d} \mathbf{x}=\sum_{\mathbf{k} \in \mathbb{N}^{d}} \frac{a_{1}^{k_{1}} \cdots a_{d}^{k_{d}}}{(|\mathbf{k}|+d) !} .
$$

We prove it by induction on $d$. For $d=1$, we have

$$
\int_{0}^{1} e^{a x} \mathrm{~d} x=\frac{e^{a}-1}{a}=\sum_{k \geq 0} \frac{a^{k}}{(k+1) !} .
$$

Let $d>1$. We write

$$
\begin{aligned}
& \int_{\Delta_{s t}} e^{a_{1} x_{1}+\cdots+a_{d} x_{d}} \mathrm{~d} \mathbf{x} \\
&=\int_{0}^{1} e^{a_{d} x_{d}}\left(\int_{\substack{x_{j} \geq 0 \\
x_{1}+\cdots+x_{d-1} \leq 1-x_{d}}} e^{a_{1} x_{1}+\cdots+a_{d-1} x_{d-1}} \mathrm{~d} x_{1} \cdots \mathrm{d} x_{d-1}\right) \mathrm{d} x_{d} .
\end{aligned}
$$

By the induction hypothesis and an obvious change of variables, the inner integral is equal to

$$
\left(1-x_{d}\right)^{d-1} \sum_{\mathbf{k} \in \mathbb{N}^{d-1}}\left(1-x_{d}\right)^{|\mathbf{k}|} \frac{a_{1}^{k_{1}} \cdots a_{d-1}^{k_{d-1}}}{(|\mathbf{k}|+d-1) !} .
$$

The result now follows from the relation

$$
\int_{0}^{1} \frac{(1-x)^{p}}{p !} e^{a x} \mathrm{~d} x=\sum_{k \geq 0} \frac{a^{k}}{(k+p+1) !} .
$$

Remark 9. Let us replace $\ell$ by $t \ell$ in (5) and expand in powers of $t$. We obtain the following formula:

$$
\int_{\Delta} \ell^{M} \mathrm{~d} m=d ! \operatorname{vol}(\Delta, \mathrm{d} m) \frac{M !}{(M+d) !} \sum_{\mathbf{k} \in \mathbb{N}^{d+1},|\mathbf{k}|=M}\left\langle\ell, \mathbf{s}_{1}\right\rangle^{k_{1}} \cdots\left\langle\ell, \mathbf{s}_{d+1}\right\rangle^{k_{d+1}}
$$


This relation is a particular case of a result of Lasserre and Avrachenkov, Proposition 18, as we will explain in Section 4.3 below.

Theorem 10. Let $\Delta$ be the simplex that is the convex hull of $(d+1)$ affinely independent vertices $\mathbf{s}_{1}, \mathbf{s}_{2}, \ldots, \mathbf{s}_{d+1}$ in $\mathbb{R}^{n}$. Then we have:

$$
\sum_{M \in \mathbb{N}} t^{M} \frac{(M+d) !}{M !} \int_{\Delta} \ell^{M} \mathrm{~d} m=d ! \operatorname{vol}(\Delta, \mathrm{d} m) \frac{1}{\prod_{j=1}^{d+1}\left(1-t\left\langle\ell, \mathbf{s}_{j}\right\rangle\right)} .
$$

Proof. We apply formula (6). Summing up from $M=0$ to $\infty$, we recognize the expansion of the right-hand side of (7) into a product of geometric series:

$$
\begin{aligned}
\sum_{M \in \mathbb{N}} t^{M} \frac{(M+d) !}{M !} & \int_{\Delta} \ell^{M} \mathrm{~d} m \\
& =d ! \operatorname{vol}(\Delta, \mathrm{d} m) \sum_{M \in \mathbb{N}} t^{M} \sum_{\mathbf{k} \in \mathbb{N}^{d+1}|, \mathbf{k}|=M}\left\langle\ell, \mathbf{s}_{1}\right\rangle^{k_{1}} \cdots\left\langle\ell, \mathbf{s}_{d+1}\right\rangle^{k_{d+1}}
\end{aligned}
$$

Theorem 10 has an extension to the integration of a product of powers of several linear forms. The following formula is implemented in our Maple program duality.mpl; see Table 7 .

Corollary 11. Let $\ell_{1}, \ldots, \ell_{D}$ be $D$ linear forms on $\mathbb{R}^{n}$. We have the following Taylor expansion:

$$
\begin{aligned}
\sum_{\mathbf{M} \in \mathbb{N}^{D}} t_{1}^{M_{1}} \cdots t_{D}^{M_{D}} \frac{(|\mathbf{M}|+d) !}{d ! \operatorname{vol}(\Delta, \mathrm{d} m)} & \int_{\Delta} \frac{\ell_{1}^{M_{1}} \cdots \ell_{D}^{M_{D}}}{M_{1} ! \cdots M_{D} !} \mathrm{d} m \\
& =\frac{1}{\prod_{i=1}^{d+1}\left(1-t_{1}\left\langle\ell_{1}, \mathbf{s}_{i}\right\rangle-\cdots-t_{D}\left\langle\ell_{D}, \mathbf{s}_{i}\right\rangle\right)} .
\end{aligned}
$$

Proof. Replace $t \ell$ with $t_{1} \ell_{1}+\cdots+t_{D} \ell_{D}$ in (7) and take the expansion in powers $t_{1}^{M_{1}} \cdots t_{D}^{M_{D}}$.

From Theorem 10, we easily obtain the "short formula" of Brion, in the case of a simplex.

Corollary 12 (Brion). Let $\Delta$ be as in the previous theorem. Let $\ell$ be a linear form which is regular w.r.t. $\Delta$, i.e., $\left\langle\ell, \mathbf{s}_{i}\right\rangle \neq\left\langle\ell, \mathbf{s}_{j}\right\rangle$ for any pair $i \neq j$. Then we have the following relations:

$$
\begin{aligned}
& \int_{\Delta} \ell^{M} \mathrm{~d} m=d ! \operatorname{vol}(\Delta, \mathrm{d} m) \frac{M !}{(M+d) !}\left(\sum_{i=1}^{d+1} \frac{\left\langle\ell, \mathbf{s}_{i}\right\rangle^{M+d}}{\prod_{j \neq i}\left\langle\ell, \mathbf{s}_{i}-\mathbf{s}_{j}\right\rangle}\right), \\
& \int_{\Delta} e^{\ell} \mathrm{d} m=d ! \operatorname{vol}(\Delta, \mathrm{d} m) \sum_{i=1}^{d+1} \frac{e^{\left\langle\ell, \mathbf{s}_{i}\right\rangle}}{\prod_{j \neq i}\left\langle\ell, \mathbf{s}_{i}-\mathbf{s}_{j}\right\rangle} .
\end{aligned}
$$

Proof. We consider the right-hand side of (7) as a rational function of $t$. The poles $t=1 /\left\langle\ell, \mathbf{s}_{i}\right\rangle$ are simple precisely when $\ell$ is regular. In this case, we obtain (9) by taking the expansion into partial fractions. The second relation follows immediately by expanding $e^{\ell}$. 
When $\ell$ is regular, Brion's formula is very short; it is a sum of $d+1$ terms. When $\ell$ is not regular, the expansion of (7) into partial fractions leads to an expression of the integral as a sum of residues. Let $K \subseteq\{1, \ldots, d+1\}$ be an index set of the different poles $t=1 /\left\langle\ell, s_{k}\right\rangle$, and for $k \in K$ let $m_{k}$ denote the order of the pole, i.e.,

$$
m_{k}=\#\left\{i \in\{1, \ldots, d+1\}:\left\langle\ell, s_{i}\right\rangle=\left\langle\ell, s_{k}\right\rangle\right\} .
$$

With this notation, we have the following formula, which is implemented in our Maple program waring.mpl; see Tables 4 and 5 .

\section{Corollary 13.}

$$
\begin{aligned}
\int_{\Delta} \ell^{M} \mathrm{~d} m & \\
& =d ! \operatorname{vol}(\Delta, \mathrm{d} m) \frac{M !}{(M+d) !} \sum_{k \in K} \operatorname{Res}_{\varepsilon=0} \frac{\left(\varepsilon+\left\langle\ell, \mathbf{s}_{k}\right\rangle\right)^{M+d}}{\varepsilon^{m_{k}} \prod_{\substack{i \in K \\
i \neq k}}\left(\varepsilon+\left\langle\ell, \mathbf{s}_{k}-\mathbf{s}_{i}\right\rangle\right)^{m_{i}}} .
\end{aligned}
$$

Remark 14. It is worth remarking that Corollaries 12 and 13 can be seen as a particular case of the localization theorem in equivariant cohomology (see for instance 8), although we did not use this fact and instead gave a simple direct calculation. In our situation, the variety is the complex projective space $\mathbb{C P}^{d}$, with action of a $d$-dimensional torus, such that the image of the moment map is the simplex $\Delta$. Brion's formula corresponds to the generic case of a one-parameter subgroup acting with isolated fixed points. In the degenerate case when the set of fixed points has components of positive dimension, the polar parts in (11) coincide with the contributions of the components to the localization formula.

A formula equivalent to Corollary 13 already appears in [3], (3.2).

\subsection{Polynomial-time algorithm for polynomial functions of a fixed num- ber of linear forms.}

Proof of Theorem 2. We now present an algorithm which, given a polynomial of the particular form $f\left(\left\langle\ell_{1}, \mathbf{x}\right\rangle, \ldots,\left\langle\ell_{D}, \mathbf{x}\right\rangle\right)$ where $f$ is a polynomial depending on a fixed number $D$ of variables, and $\left\langle\ell_{j}, \mathbf{x}\right\rangle=L_{j 1} x_{1}+\cdots+L_{j n} x_{n}$ for $j=1, \ldots, D$ are linear forms on $\mathbb{R}^{n}$, computes its integral on a simplex, in time polynomial on the input data. This algorithm relies on Corollary 11.

The number of monomials of degree $M$ in $D$ variables is equal to $\left(\begin{array}{c}M+D-1 \\ D-1\end{array}\right)$. Therefore, when $D$ is fixed, the number of monomials of degree at most $M$ in $D$ variables is $\mathrm{O}\left(M^{D}\right)$. When the number of variables $D$ of a straight-line program $\Phi$ is fixed, it is possible to compute a sparse or dense representation of the polynomial represented by $\Phi$ in polynomial time, by a straightforward execution of the program. Indeed, all intermediate results can be stored as sparse or dense polynomials with $\mathrm{O}\left(M^{D}\right)$ monomials. Since the program $\Phi$ is single-intermediate-use, the binary encoding size of all coefficients of the monomials can be bounded polynomially by the input encoding size. Thus it is enough to compute the integral of a monomial,

$$
\int_{\Delta}\left\langle\ell_{1}, \mathbf{x}\right\rangle^{M_{1}} \cdots\left\langle\ell_{D}, \mathbf{x}\right\rangle^{M_{D}} \mathrm{~d} m
$$

From Corollary 11, it follows that

$$
\frac{(|\mathbf{M}|+d) !}{d ! \operatorname{vol}(\Delta, \mathrm{d} m)} \int_{\Delta} \frac{\ell_{1}^{M_{1}} \cdots \ell_{D}^{M_{D}}}{M_{1} ! \cdots M_{D} !} \mathrm{d} m
$$


is the coefficient of $t_{1}^{M_{1}} \cdots t_{D}^{M_{D}}$ in the Taylor expansion of

$$
\frac{1}{\prod_{i=1}^{d+1}\left(1-t_{1}\left\langle\ell_{1}, \mathbf{s}_{i}\right\rangle-\cdots-t_{D}\left\langle\ell_{D}, \mathbf{s}_{i}\right\rangle\right)} .
$$

Since $D$ is fixed, this coefficient can be computed in time polynomial with respect to $\mathbf{M}$ and the input data, by multiplying the series truncated at degree $|\mathbf{M}|$, as explained in Lemma 4

Finally, $\operatorname{vol}(\Delta, \mathrm{d} m)$ needs to be computed. If $\Delta=\operatorname{conv}\left\{\mathbf{s}_{1}, \ldots, \mathbf{s}_{d+1}\right\}$ is fulldimensional $(d=n)$, we can do so by computing the determinant of the matrix formed by difference vectors of the vertices:

$$
\operatorname{vol}(\Delta, \mathrm{d} m)=\frac{1}{n !}\left|\operatorname{det}\left(\mathbf{s}_{1}-\mathbf{s}_{n+1}, \ldots, \mathbf{s}_{n}-\mathbf{s}_{n+1}\right)\right| .
$$

If $\Delta$ is lower-dimensional, we first compute a basis $B \in \mathbb{Z}^{n \times d}$ of the intersection lattice $\Lambda=\operatorname{lin}(\Delta) \cap \mathbb{Z}^{n}$. This can be done in polynomial time by applying an efficient algorithm for computing the Hermite normal form 24. Then we express each difference vector $\mathbf{v}_{i}=\mathbf{s}_{i}-\mathbf{s}_{d+1} \in \operatorname{lin}(\Delta)$ for $i=1, \ldots, d$ using the basis $B$ as $\mathbf{v}_{i}=B \mathbf{v}_{i}^{\prime}$, where $\mathbf{v}_{i} \in \mathbb{Q}^{d}$. We obtain

$$
\operatorname{vol}(\Delta, \mathrm{d} m)=\frac{1}{d !}\left|\operatorname{det}\left(\mathbf{v}_{1}^{\prime}, \ldots, \mathbf{v}_{d}^{\prime}\right)\right|,
$$

thus the volume computation is reduced to the calculation of a determinant. This finishes the proof of Theorem 2 .

3.4. Polynomial time algorithms for polynomials of fixed degree. In the present section, we assume that the total degree of the input polynomial $f$ we wish to integrate is a constant $M$.

Proof of Corollary 3. First of all, when the formal degree $M$ of a straight-line program $\Phi$ is fixed, it is possible to compute a sparse or dense representation of the polynomial represented by $\Phi$ in polynomial time, by a straightforward execution of the program. Indeed, all intermediate results can be stored as sparse or dense polynomials with $\mathrm{O}\left(n^{M}\right)$ monomials. Since the program $\Phi$ is single-intermediateuse, the binary encoding size of all coefficients of the monomials can be bounded polynomially by the input encoding size.

Now, the key observation is that a monomial of degree at most $M$ depends effectively on $D \leq M$ variables $x_{i_{1}}, \ldots, x_{i_{D}}$. Thus it is of the form

$$
\ell_{1}^{M_{1}} \cdots \ell_{D}^{M_{D}}
$$

where the linear forms $\ell_{j}(\mathbf{x})=x_{i_{j}}$ are the coordinates that effectively appear in the monomial. Thus, Corollary 3 follows immediately from Theorem 2. This method is implemented in our Maple program duality.mpl, see Tables 7 and 131.

Remark 15. The relations in Corollary 12 can be interpreted as equalities between meromorphic functions of $\ell$. The right-hand side is a sum of meromorphic functions whose poles cancel out so that the sum is actually analytic. We derive from this another polynomial time algorithm for computating the integral

$$
\int_{\Delta} x_{1}^{m_{1}} \cdots x_{d}^{m_{d}} \mathrm{~d} m
$$

\footnotetext{
${ }^{1}$ See the "Note" following $\S 6$ regarding Tables $9-16$.
} 
More precisely, let us write $\langle\ell, \mathbf{x}\rangle=y_{1} x_{1}+\cdots+y_{d} x_{d}$. Then the integral $\int_{\Delta} x_{1}^{m_{1}} \cdots$ $x_{d}^{m_{d}} \mathrm{~d} m$ is the coefficient of $\frac{y_{1}^{m_{1}} \cdots y_{d}^{m_{d}}}{m_{1} ! \cdots m_{d} !}$ in the Taylor expansion of $\int_{\Delta} e^{y_{1} x_{1}+\cdots+y_{d} x_{d}} \mathrm{~d} m$. We compute it by taking the expansion of each of the terms of the right hand-side of (9) into an iterated Laurent series with respect to the variables $x_{1}, \ldots, x_{d}$. This method is implemented in our Maple program iterated-laurent.mpl; see Tables 12 and 14.

In the following, we give another proof of Corollary 3 , based on decompositions of polynomials as sums of powers of linear forms.

Alternative proof of Corollary [3. From Corollaries 12 and 13, we derive another efficient algorithm, as follows. The key idea now is that one can decompose the polynomial $f$ as a sum $f:=\sum_{\ell} c_{\ell} \ell_{j}^{M}$ with at most $2^{M}$ terms in the sum. We use the well-known identity

$$
\begin{aligned}
& x_{1}^{M_{1}} x_{2}^{M_{2}} \cdots x_{n}^{M_{n}} \\
= & \frac{1}{|\mathbf{M}| !} \sum_{0 \leq p_{i} \leq M_{i}}(-1)^{|\mathbf{M}|-\left(p_{1}+\cdots+p_{n}\right)}\left(\begin{array}{c}
M_{1} \\
p_{1}
\end{array}\right) \cdots\left(\begin{array}{c}
M_{n} \\
p_{n}
\end{array}\right)\left(p_{1} x_{1}+\cdots+p_{n} x_{n}\right)^{|\mathbf{M}|},
\end{aligned}
$$

where $|\mathbf{M}|=M_{1}+\cdots+M_{n} \leq M$.

In the implementation of this method, we may group together proportional linear forms. The number $F(n, M)$ of primitive vectors $\left(p_{1}, \ldots, p_{n}\right)$ which appear in the decomposition of a polynomial of total degree $\leq M$ is given by the following closed formula 2

Lemma 16. Let

$$
F(n, M)=\operatorname{Card}\left(\left\{\left(p_{1}, \ldots, p_{n}\right) \in \mathbb{N}^{n}, \operatorname{gcd}\left(p_{1}, \ldots, p_{n}\right)=1,1 \leq \sum_{i} p_{i} \leq M\right\}\right) .
$$

Then

$$
F(n, M)=\sum_{d=1}^{M} \mu(d)\left(\left(\begin{array}{c}
n+\left[\frac{M}{d}\right] \\
n
\end{array}\right)-1\right),
$$

where $\mu(d)$ is the Möbius function.

When $M$ is fixed and $n \rightarrow \infty$, we have

$$
F(n, M)=\frac{n^{M}}{M !}+\mathrm{O}\left(n^{M-1}\right) .
$$

Proof. Let $G(n, M)=\operatorname{Card}\left(\left\{\left(p_{1}, \ldots, p_{n}\right) \in \mathbb{N}^{n}, 1 \leq \sum p_{i} \leq M\right\}\right)$. By grouping together the vectors $\left(p_{1}, \ldots, p_{n}\right)$ with a given gcd $d$, we obtain

$$
G(n, M)=\sum_{d=1}^{M} F\left(n,\left[\frac{M}{d}\right]\right) .
$$

Moreover, the number of all integral vectors $\left(p_{1}, \ldots, p_{n}\right) \in \mathbb{N}^{n}$ such that $\sum_{i} p_{i} \leq M$ is equal to the binomial coefficient $\left(\begin{array}{c}n+M \\ n\end{array}\right)$. When we omit the zero-vector we obtain

$$
G(n, M)=\left(\begin{array}{c}
n+M \\
n
\end{array}\right)-1 .
$$

\footnotetext{
${ }^{2}$ Lemma 16 was kindly supplied by Christophe Margerin.
} 
Then we obtain (14) by applying the second form of the Möbius inversion formula. The asymptotics follow easily from (14).

Thus formula (13), together with Corollary 13, give another polynomial time algorithm for integrating a polynomial of fixed degree. It is implemented in our Maple program waring.mpl; see Tables 5 and 11.

The problem of finding a decomposition with the smallest possible number of summands is known as the polynomial Waring problem. Alexander and Hirschowitz [1] solved the generic problem (see [1] for an extensive survey).

Theorem 17. The smallest integer $r(M, n)$, such that a generic homogeneous polynomial of degree $M$ in $n$ variables is expressible as the sum of $r(M, n) M$-th powers of linear forms, is given by

$$
r(M, n)=\left\lceil\frac{\left(\begin{array}{c}
n+M-1 \\
M
\end{array}\right)}{n}\right\rceil,
$$

with the exception of the cases $r(3,5)=8, r(4,3)=6, r(4,4)=10, r(4,5)=15$, and $M=2$, where $r(2, n)=n$.

An algorithm for decomposing a given polynomial into the smallest possible number of powers of linear forms can be found in [10.

In the extreme case, when the polynomial $f$ happens to be the power of one linear form $\ell$, one should certainly avoid applying the above decomposition formula to each of the monomials of $f$. We remark that, when the degree is fixed, we can decide in polynomial time whether a polynomial $f$, given in sparse or dense monomial representation, is a power of a linear form $\ell$ and, if so, construct such a linear form.

\section{OTHER ALGORITHMS FOR INTEGRATION AND EXTENSIONS TO OTHER POLYTOPES}

We conclude with a discussion of how to extend integration to other polytopes and a review of the complexity of other methods to integrate polynomials over polytopes.

4.1. A formula of Lasserre-Avrachenkov. Another nice formula is the Lasserre-Avrachenkov formula for the integration of a homogeneous polynomial 27 on a simplex. As we explain below, this yields a polynomial-time algorithm for the problem of integrating a polynomial of fixed degree over a polytope in varying dimension, thus providing an alternative proof of 3 .

Proposition 18 (27]). Denote by $\mathrm{d} \mathbf{x}$ the standard Lebesgue measure. Let $H$ be a symmetric multilinear form defined on $\left(\mathbb{R}^{d}\right)^{M}$. Let $\mathbf{s}_{1}, \mathbf{s}_{2}, \ldots, \mathbf{s}_{d+1}$ be the vertices of a d-dimensional simplex $\Delta$. Then one has

$$
\int_{\Delta} H(\mathbf{x}, \mathbf{x}, \ldots, \mathbf{x}) d \mathbf{x}=\frac{\operatorname{vol}(\Delta)}{\left(\begin{array}{c}
M+d \\
M
\end{array}\right)} \sum_{1 \leq i_{1} \leq i_{2} \leq \cdots \leq i_{M} \leq d+1} H\left(\mathbf{s}_{i_{1}}, \mathbf{s}_{i_{2}}, \ldots, \mathbf{s}_{i_{M}}\right) .
$$


Remark 19. By reindexing the summation in (15), as $H$ is symmetric, we obtain

$$
\begin{array}{rl}
\int_{\Delta} H & H(\mathbf{x}, \mathbf{x}, \ldots, \mathbf{x}) d \mathbf{x} \\
& =\frac{\operatorname{vol}(\Delta)}{\left(\begin{array}{c}
M+d \\
M
\end{array}\right)} \sum_{k_{1}+\cdots+k_{d+1}=M} H\left(\mathbf{s}_{1}, \ldots, \mathbf{s}_{1}, \ldots, \mathbf{s}_{d+1}, \ldots, \mathbf{s}_{d+1}\right),
\end{array}
$$

where $\mathbf{s}_{1}$ is repeated $k_{1}$ times, $\mathbf{s}_{2}$ is repeated $k_{2}$ times, etc. When $H$ is of the form $H=\prod_{i=1}^{M}\left\langle\ell, \mathbf{x}_{i}\right\rangle$, for a single linear form $\ell$, then (16) coincides with (6) in Remark 9 .

Now any polynomial $f$ which is homogeneous of degree $M$ can be written as $f(\mathbf{x})=H_{f}(\mathbf{x}, \mathbf{x}, \ldots, \mathbf{x})$ for a unique multilinear form $H_{f}$. If $f=\ell^{M}$, then $H_{f}=\prod_{i=1}^{M}\left\langle\ell, \mathbf{x}_{i}\right\rangle$. Thus for fixed $M$ the computation of $H_{f}$ can be achieved by decomposing $f$ into a linear combination of powers of linear forms, as we did in the proof of Corollary 3. Alternatively one can use the well-known polarization formula,

$$
H_{f}\left(\mathbf{x}_{1}, \ldots, \mathbf{x}_{M}\right)=\frac{1}{2^{M} M !} \sum_{\varepsilon \in\{ \pm 1\}^{M}} \varepsilon_{1} \varepsilon_{2} \cdots \varepsilon_{M} f\left(\sum_{i=1}^{M} \varepsilon_{i} \mathbf{x}_{i}\right),
$$

Thus from (15) we get the following corollary.

Corollary 20. Let $f$ be a homogeneous polynomial of degree $M$ in $d$ variables, and let $\mathbf{s}_{1}, \mathbf{s}_{2}, \ldots, \mathbf{s}_{d+1}$ be the vertices of a d-dimensional simplex $\Delta$. Then

$$
\begin{aligned}
& \int_{\Delta} f(\mathbf{y}) \mathrm{d} \mathbf{y} \\
& \quad=\frac{\operatorname{vol}(\Delta)}{2^{M} M !\left(\begin{array}{c}
M+d \\
M
\end{array}\right)} \sum_{1 \leq i_{1} \leq i_{2} \leq \cdots \leq i_{M} \leq d+1} \sum_{\varepsilon \in\{ \pm 1\}^{M}} \varepsilon_{1} \varepsilon_{2} \cdots \varepsilon_{M} f\left(\sum_{k=1}^{M} \varepsilon_{k} \mathbf{s}_{i_{k}}\right) .
\end{aligned}
$$

We remark that when we fix the degree $M$ of the homogeneous polynomial $f$, the length of the polarization formula (thus the length of the second sum in (18)) is a constant. The length of the first sum in (18) is $\mathrm{O}\left(n^{M}\right)$. Thus, for fixed degree in varying dimension, we obtain another polynomial-time algorithm for integrating over a simplex. Note that the integral resembles a cubature formula, as the integral is a weighted sum (with weights $+1,-1$ ) of $f$ evaluated at sums of vertices. We will discuss more about this in Section 5 .

4.2. Traditional conversion of the integral as iterated univariate integrals. Let $P \subseteq \mathbb{R}^{d}$ be a full-dimensional polytope and $f$ a polynomial. The traditional method with which we teach our calculus students to compute multivariate integrals over a bounded region requires them to write the integral $\int_{P} f \mathrm{~d} m$ as a sum of sequences of one-dimensional integrals

$$
\sum_{j=1}^{K} \int_{a_{1 j}}^{b_{1 j}} \int_{a_{2 j}}^{b_{2 j}} \cdots \int_{a_{d j}}^{b_{d j}} f \mathrm{~d} x_{i_{1}} \mathrm{~d} x_{i_{2}} \ldots \mathrm{d} x_{i_{d}}
$$

for which we know the limits of integration $a_{i j}, b_{i j}$ explicitly. The problem of finding the limits of integration and the sum has interesting complexity related to the wellknown Fourier-Motzkin elimination method (see Chapter One in [37] for a short introduction). 
Given a system of linear inequalities $A \mathbf{x} \leq \mathbf{b}$, describing a polytope $P \subset \mathbb{R}^{d}$, Fourier-Motzkin elimination is an algorithm that computes a new larger system of inequalities $\hat{A} \mathbf{x} \leq \hat{\mathbf{b}}$ with the property that those inequalities that do not contain the variable $x_{d}$ describe the projection of $P$ into the hyperplane $x_{d}=0$. We will not explain the details, but Fourier-Motzkin elimination is quite similar to Gaussian elimination in the sense that the main operations necessary to eliminate the last variable $x_{d}$ require one to rearrange, scale, and add rows of the matrix $(A, \mathbf{b})$, but unlike Gaussian elimination, new inequalities are added to the system.

It was first observed by Schechter 35] that Fourier-Motzkin elimination provides a way to generate the traditional iterated integrals. More precisely, let us call $P_{d}$ the projection of $P$ into the the hyperplane $x_{d}=0$. Clearly, when integrating over a polytopal region we expect that the limits of integration will be affine functions. From the output of Fourier-Motzkin $\hat{A} \mathbf{x} \leq \hat{\mathbf{b}}$, we have that $\mathbf{x} \in P$ if and only if $\left(x_{1}, \ldots, x_{d-1}\right) \in P_{d}$, and for the first $k+r$ inequalites of the system

$$
x_{d} \leq \hat{b}_{i}-\sum_{j=1}^{d-1} \hat{a}_{i j} x_{j}=A_{i}^{\mathrm{u}}\left(x_{1}, \ldots, x_{d-1}\right)
$$

for $i=1, \ldots, k$, as well as

$$
x_{d} \geq \hat{b}_{k+i}-\sum_{j=1}^{d-1} \hat{a}_{k+i j} x_{j}=A_{i}^{\mathrm{l}}\left(x_{1}, \ldots, x_{d-1}\right)
$$

for $i=1, \ldots, r$. Then, if we define

$$
m\left(x_{1}, \ldots, x_{d}\right)=\max \left\{A_{j}^{1}\left(x_{1}, \ldots, x_{d-1}\right), j=1, \ldots, r\right\}
$$

and

$$
M\left(x_{1}, \ldots, x_{d}\right)=\min \left\{A_{j}^{\mathrm{u}}\left(x_{1}, \ldots, x_{d-1}\right), j=1, \ldots, r\right\}
$$

we can write

$$
\int_{P} f(\mathbf{x}) \mathrm{d} m=\int_{P_{d}} \int_{m}^{M} f(\mathbf{x}) \mathrm{d} x_{1} \mathrm{~d} x_{2} \cdots \mathrm{d} x_{d} .
$$

Finally the convex polytope $P_{d}$ can be decomposed into polyhedral regions where the functions $m, M$ become simply affine functions from among the list. Since the integral is additive we get an expression

$$
\int_{P} f(\mathbf{x}) \mathrm{d} m=\sum_{i, j} \int_{P_{d}^{i j}} \int_{A_{j}^{1}}^{A_{j}^{\mathrm{u}}} f(\mathbf{x}) \mathrm{d} x_{1} \mathrm{~d} x_{2} \cdots \mathrm{d} x_{d} .
$$

Finally, by repeating the elimination of variables we recover the full iterated list in (19). As it was observed in [35], this algorithm is unfortunately not efficient because the iterated Fourier-Motzkin elimination procedure can produce exponentially many inequalities for the description of the projection (when the dimension $d$ varies). Thus the number of summands considered can in fact grow exponentially.

4.3. Two formulas for integral computation. We would like to review two formulas that are nice and could speed up computation in particular cases, although they do not seem to yield efficient algorithms just on their own.

First, one may reduce the computation of $\int_{P} f \mathrm{~d} m$ to integrals on the facets of $P$ by applying the Stokes formula. We must be careful to use a rational Lebesgue measure on each facet. As shown in 4], we have the following result. 
Theorem 21. Let $\left\{F_{i}\right\}_{i=1, \ldots, m}$ be the set of facets of a full-dimensional polytope $P \subseteq \mathbb{R}^{n}$. For each $i$, let $\mathbf{n}_{i}$ be a rational vector which is transverse to the facet $F_{i}$ and pointing outwards $P$, and let $\mathrm{d} \mu_{i}$ be the Lebesgue measure on the affine hull of $F_{i}$ which is defined by contracting the standard volume form of $\mathbb{R}^{n}$ with $\mathbf{n}_{i}$. Then

$$
I_{P}(\mathbf{a})=\int_{P} e^{\langle\mathbf{a}, \mathbf{x}\rangle} \mathrm{d} \mathbf{x}=\frac{1}{\langle\mathbf{a}, \mathbf{y}\rangle} \sum_{i=1}^{m}\left\langle\mathbf{n}_{i}, \mathbf{y}\right\rangle \int_{F_{i}} e^{\langle\mathbf{a}, \mathbf{x}\rangle} \mathrm{d} \mu_{i}
$$

for all $\mathbf{a} \in \mathbb{C}^{n}$ and $\mathbf{y} \in \mathbb{R}^{n}$ such that $\langle\mathbf{y}, \mathbf{a}\rangle \neq 0$.

It is clear that, by considering the expansion of the analytic function $\int_{P} e^{\langle\mathbf{a}, \mathbf{x}\rangle} \mathrm{d} \mathbf{x}$, we can again obtain an analogous result for polynomials. An alternative proof was provided by [26. The above theorem, however, does not necessarily reduce the computational burden because, depending on the representation of the polytope, the number of facets can be large, and also the facets themselves can be complicated polytopes. Yet, together with our results we obtain the following corollary for two special cases.

Corollary 22. There is a polynomial-time algorithm for the following problem. Input:

$\left(\mathrm{I}_{1}\right)$ the dimension $n \in \mathbb{N}$ in unary encoding,

$\left(\mathrm{I}_{2}\right)$ a list of rational vectors in binary encoding, namely

(i) either vectors $\left(\mathbf{h}_{1}, h_{1,0}\right), \ldots,\left(\mathbf{h}_{m}, h_{m, 0}\right) \in \mathbb{Q}^{n+1}$ that describe the facetdefining inequalities $\left\langle\mathbf{h}_{i}, \mathbf{x}\right\rangle \leq h_{i, 0}$ of a simplicial full-dimensional rational polytope $P$ (i.e. all its faces are simplices),

(ii) or vectors $\mathbf{s}_{1}, \ldots, \mathbf{s}_{N} \in \mathbb{Q}^{n}$ that are the vertices of a simple fulldimensional rational polytope $P$,

$\left(\mathrm{I}_{3}\right)$ a rational vector $\mathbf{a} \in \mathbb{Q}^{n}$ in binary encoding,

$\left(\mathrm{I}_{4}\right)$ an exponent $M \in \mathbb{N}$ in unary encoding.

Output, in binary encoding:

$\left(\mathrm{O}_{1}\right)$ the rational number

$$
\int_{P} f(\mathbf{x}) \mathrm{d} m, \text { where } f(\mathbf{x})=\langle\mathbf{a}, \mathbf{x}\rangle^{M},
$$

where $\mathrm{d} m$ is the standard Lebesgue measure on $\mathbb{R}^{n}$.

Proof. In the case (i) of simplicial polytopes $P$ given by facet-defining inequalities, we can use linear programming to compute in polynomial time a $V$-representation for each simplex $F_{i}$ that is a facet of $P$. By applying Theorem 21 with $t \mathbf{a}$ in place of $\mathbf{a}$ and extracting the coefficient of $t^{M}$ in the Taylor expansion of the analytic function $t \mapsto I_{P}(t \mathbf{a})$, we obtain the formula

$$
\int_{P}\langle\mathbf{a}, \mathbf{x}\rangle^{M} \mathrm{~d} \mathbf{x}=\frac{1}{(M+1)\langle\mathbf{y}, \mathbf{a}\rangle} \sum_{i=1}^{m}\left\langle\mathbf{y}, \mathbf{n}_{i}\right\rangle \int_{F_{i}}\langle\mathbf{a}, \mathbf{x}\rangle^{M+1} \mathrm{~d} \mu_{i}
$$

which holds for all $\mathbf{y} \in \mathbb{R}^{n}$ with $\langle\mathbf{y}, \mathbf{a}\rangle \neq 0$. It is known that a suitable $\mathbf{y} \in \mathbb{Q}^{n}$ can be constructed in polynomial time. The integrals on the right-hand side can now be evaluated in polynomial time using Theorem 2

In the case (ii) of simple polytopes $P$ given by their vertices, we make use of the fact that a variant of Brion's formula (9) actually holds for arbitrary rational polytopes. For a simple polytope $P$, it takes the following form (collecting terms 
in the Taylor expansion of $e^{t \ell}$, the formula is a consequence from Proposition 3.10 in [14]):

$$
\int_{P} \ell^{M} \mathrm{~d} x=\frac{M !}{(M+n) !} \sum_{i=1}^{N} \Delta_{i} \frac{\left\langle\ell, \mathbf{s}_{i}\right\rangle^{M+n}}{\prod_{\mathbf{s}_{j} \in N\left(\mathbf{s}_{i}\right)}\left\langle\ell, \mathbf{s}_{i}-\mathbf{s}_{j}\right\rangle},
$$

where $N\left(\mathbf{s}_{i}\right)$ denotes the set of vertices adjacent to $\mathbf{s}_{i}$ in $P$, and

$$
\Delta_{i}=\left|\operatorname{det}\left(\mathbf{s}_{i}-\mathbf{s}_{j}\right)_{j \in N\left(\mathbf{s}_{i}\right)}\right| .
$$

The right-hand side is a sum of rational functions of $\ell$, where the denominators cancel out so that the sum is actually polynomial. If $\ell$ is regular, that is to say $\left\langle\ell, \mathbf{s}_{i}-\mathbf{s}_{j}\right\rangle \neq 0$ for any $i$ and $j \in N\left(\mathbf{s}_{i}\right)$, then the integral can be computed by (20), which is a very short formula. However, it becomes difficult to extend the method which we used in the case of a simplex. Instead, we can do a perturbation. In (20), we replace $\ell$ by $\ell+\varepsilon \ell^{\prime}$, where $\ell^{\prime}$ is such that $\ell+\varepsilon \ell^{\prime}$ is regular for $\varepsilon \neq 0$. The algorithm for choosing $\ell^{\prime}$ is bounded polynomially. Then we do expansions in powers of $\varepsilon$ as explained in Lemma 4 .

4.4. Connections to triangulations and splines. It is well known that any convex polytope can be triangulated into finitely many simplices. Thus we can use our result to extend the integration of polynomials over any convex polytope. The complexity of doing it this way will directly depend on the number of simplices in a triangulation. This raises the issue of finding the smallest triangulation possible of a given polytope. Unfortunately this problem was proved to be NP-hard even for fixed dimension three (see [18]). Thus it is in general not a good idea to spend time finding the smallest triangulation possible. A cautionary remark is that one can naively assume that triangulations help for non-convex polyhedral regions, while in reality it does not because there exist non-convex polyhedra that are not triangulable unless one adds new points. Deciding how many new points are necessary is an NP-hard problem [18].

Another interesting approach to the integration of polynomials over polytopes is through the theory of B-splines and multivariate truncated powers. B-splines are closely related to the volume of polytopes (see Chapter 4 of [32]). Recently Xu 36] has observed that the integration of monomials over polytopes is equivalent to the computation of the multivariate truncated power of a matrix $T(\mathbf{x} \mid M)$ (this integral operator on matrices was introduced by Dahmen in [17]). More precisely, for every $\mathbf{k}=\left(k_{1}, \ldots, k_{n}\right) \in \mathbb{Z}_{+}^{n}$ and an $n$-column matrix $M=\left(\mathbf{m}_{1}, \ldots, \mathbf{m}_{n}\right)$ we set

$$
M^{\mathbf{k}}:=(\underbrace{\mathbf{m}_{1}, \ldots, \mathbf{m}_{1}}_{k_{1}+1}, \underbrace{\mathbf{m}_{2}, \ldots, \mathbf{m}_{2}}_{k_{2}+1}, \ldots, \underbrace{\mathbf{m}_{n}, \ldots, \mathbf{m}_{n}}_{k_{n}+1}) .
$$

Theorem 23. Suppose $\mathbf{k}=\left(k_{1}, \ldots, k_{n}\right) \in \mathbb{Z}_{+}^{n}$ and gives a monomial $f(\mathbf{u})=$ $\prod_{j=1}^{n} u_{j}^{k_{j}}$. Let $P:=\left\{\mathbf{u} \in \mathbb{R}_{+}^{n} \mid M \mathbf{u}=\mathbf{x}\right\}$ be a polyhedron. Then

$$
T\left(\mathbf{x} \mid M^{\mathbf{k}}\right)=\frac{1}{\mathbf{k} ! \cdot \sqrt{\operatorname{det}\left(M M^{T}\right)}} \int_{P} f(\mathbf{u}) \mathrm{d} \mathbf{u},
$$

where $\mathbf{k} !:=k_{1} ! \cdots k_{n} !$

Such an approach yields a polynomial-time algorithm when the number of variables is fixed. 


\section{IMPLEMENTATION AND COMPUTATIONAL EXPERIMENTS}

We have written Maple programs in order to perform some initial experiments with the three methods described in Section 3.4. The programs are available at [2] 3

\subsection{Integration of a power of a linear form and decomposition of poly- nomials into powers of linear forms.}

5.1.1. Decomposition of polynomials into powers of linear forms. Table 9 shows the number $F(n, M)$ of primitive linear forms $\left(p_{1}, \ldots, p_{n}\right)$ which may appear in the decomposition (13) of a polynomial of total degree $\leq M$. This number is computed using the closed formula (14).

5.1.2. Integration of a power of a linear form over a simplex. We have written a Maple program which implements the method of Corollary 13 for the efficient integration of a power of one linear form over a simplex, $\int_{\Delta} \ell^{M} \mathrm{~d} m 4$ In a computational experiment, for a given dimension $n$ and degree $M$ we picked random full-dimensional simplices $\Delta$ and random linear forms $\ell$ and used the Maple program to compute the integral. Table 4 shows the computation times 5

TABLE 4. Integration of powers of linear forms over simplices

\begin{tabular}{rrrrrrrr}
\hline & \multicolumn{8}{c}{ Degree $M$} \\
\cline { 2 - 8 }$n$ & 2 & 10 & 20 & 50 & 100 & 300 & 1000 \\
\hline 10 & 0.0 & 0.0 & 0.0 & 0.1 & 0.0 & 0.0 & 0.0 \\
20 & 0.1 & 0.2 & 0.1 & 0.2 & 0.1 & 0.2 & 0.2 \\
50 & 1.0 & 1.4 & 1.4 & 1.6 & 1.6 & 1.6 & 1.7 \\
100 & 5.1 & 8.4 & 8.7 & 9.2 & 9.5 & 10.0 & 11.0 \\
200 & 36 & 71 & 84 & 88 & 97 & 110 & 120 \\
300 & 150 & 320 & 400 & 470 & 520 & 530 & \\
400 & 500 & & & & & & \\
1000 & & & & & & & \\
\hline
\end{tabular}

5.1.3. Integration of a monomial over a simplex by decomposition as a sum of powers of linear forms. Next, we tested the algorithm which computes the integral of a monomial $\mathbf{x}^{\mathbf{M}}$ over a simplex $\Delta$, by decomposing it as a sum of powers of linear forms. This algorithm was discussed in Section 3.4. In our experiments, for given dimension $n$ and total degree $M$, we picked 50 combinations of a random simplex $\Delta$ of dimension $n$ and a random exponent vector $\mathbf{M}=\left(M_{1}, \ldots, M_{n}\right)$ with $\sum_{i=1}^{n} M_{i}=M$.

\footnotetext{
${ }^{3}$ All algorithms are implemented in the files waring.mpl, iterated_laurent.mpl and duality.mpl; all tables with random examples are created using procedures in examples.mpl and tables.mpl.

${ }^{4}$ The integration is done by the Maple procedure integral_power_linear_form in waring.mpl.

${ }^{5}$ All experiments were done with Maple 12 on Sun Fire V440 machines with UltraSPARC-IIIi processors running at $1.6 \mathrm{GHz}$. The computation times are given in CPU seconds. All experiments were subject to a time limit of 600 seconds per example.
} 
TABLE 5. Integration of a random monomial of prescribed degree by decomposition into a sum of powers of linear forms

\begin{tabular}{|c|c|c|c|c|c|c|c|c|c|c|c|}
\hline \multirow[b]{2}{*}{$n$} & \multicolumn{11}{|c|}{ Degree } \\
\hline & 1 & 2 & 5 & 10 & 20 & 30 & 40 & 50 & 100 & 200 & 300 \\
\hline \multirow[t]{3}{*}{2} & 0 & 0 & 0 & 0 & 0 & 0 & 0.1 & 0.3 & 0.8 & 6.2 & 0.3 \\
\hline & 0 & 0 & 0 & 0.1 & 0.2 & 0.5 & 1.0 & 1.5 & 8.3 & 38 & 90 \\
\hline & 0 & 0.1 & 0.1 & 0.1 & 0.4 & 0.8 & 1.9 & 2.6 & 12 & 59 & 174 \\
\hline \multirow[t]{3}{*}{3} & 0 & 0 & 0 & 0 & 0 & 0.4 & 0.5 & 0.9 & 4.6 & & \\
\hline & 0 & 0 & 0 & 0.2 & 1.1 & 3.0 & 7.4 & 17 & 173 & & \\
\hline & 0 & 0.2 & 0.1 & 0.4 & 2.2 & 7.0 & 19 & 37 & 440 & & \\
\hline \multirow[t]{3}{*}{4} & 0 & 0 & 0 & 0.1 & 0.6 & 1.5 & 4.8 & 9.7 & & & \\
\hline & 0 & 0 & 0.1 & 0.4 & 3.6 & 15 & 52 & 135 & & & \\
\hline & 0 & 0.2 & 0.2 & 0.9 & 8.7 & 44 & 149 & 404 & & & \\
\hline \multirow[t]{2}{*}{5} & 0 & 0 & 0 & 0.1 & 0.3 & 4.5 & & & & & \\
\hline & $\begin{array}{l}0 \\
0\end{array}$ & $\begin{array}{l}\mathbf{O}_{2} \\
0_{2}\end{array}$ & $\begin{array}{l}\mathbf{0 . 1} \\
0.3\end{array}$ & $\begin{array}{l}\mathbf{0 . 7} \\
1.9\end{array}$ & $\begin{array}{r}8.8 \\
27\end{array}$ & $\begin{array}{r}\mathbf{4 8} \\
195\end{array}$ & & & & & \\
\hline \multirow{3}{*}{6} & & 0 & 0 & 0.2 & 13 & & & & & & \\
\hline & 0 & 0 & 0.2 & 1.3 & 24 & 144 & & & & & \\
\hline & 0.2 & 0.1 & 0.4 & 2.7 & 74 & & & & & & \\
\hline \multirow[t]{3}{*}{7} & 0 & 0 & 0 & 0.5 & 5.9 & & & & & & \\
\hline & 0 & 0 & 0.3 & 2.1 & 53 & & & & & & \\
\hline & 0.2 & 0.1 & 0.6 & 5.0 & 152 & & & & & & \\
\hline \multirow[t]{3}{*}{8} & 0 & 0 & 0.1 & 0.4 & 11 & & & & & & \\
\hline & 0 & 0 & 0.3 & 3.2 & 72 & & & & & & \\
\hline & 0.2 & 0.2 & 0.6 & 8.5 & 216 & & & & & & \\
\hline \multirow[t]{2}{*}{10} & 0 & 0 & 0.2 & 1.5 & & & & & & & \\
\hline & $\begin{array}{l}\mathbf{0} \\
0.3\end{array}$ & $\begin{array}{l}0.1 \\
0.2\end{array}$ & $\begin{array}{l}0.4 \\
0.8\end{array}$ & $\begin{array}{l}6.1 \\
12\end{array}$ & & & & & & & \\
\hline \multirow[t]{3}{*}{15} & 0 & 0 & 0.3 & 3.8 & & & & & & & \\
\hline & 0.1 & 0.1 & 1.2 & 17 & & & & & & & \\
\hline & 0.2 & 0.3 & 1.8 & 41 & & & & & & & \\
\hline \multirow[t]{3}{*}{20} & 0.1 & 0.1 & 0.6 & 4.4 & & & & & & & \\
\hline & 0.1 & 0.3 & 2.2 & 41 & & & & & & & \\
\hline & 0.2 & 0.4 & 2.9 & 73 & & & & & & & \\
\hline \multirow[t]{3}{*}{30} & 0.1 & 0.2 & 2.7 & 37 & & & & & & & \\
\hline & 0.2 & 0.5 & 5.1 & 106 & & & & & & & \\
\hline & & & 6.8 & & & & & & & & \\
\hline \multirow{2}{*}{40} & 0.3 & 0.3 & 5.2 & 93 & & & & & & & \\
\hline & $\begin{array}{l}\mathbf{0 . 4} \\
0.6\end{array}$ & $\begin{array}{l}\mathbf{1 . 1} \\
1.3\end{array}$ & 10 & 242 & & & & & & & \\
\hline \multirow[t]{3}{*}{50} & 0.5 & 0.7 & 8.2 & & & & & & & & \\
\hline & 0.6 & 1.8 & 17 & & & & & & & & \\
\hline & 0.8 & 2.0 & 20 & & & & & & & & \\
\hline
\end{tabular}

First we decompose a given monomial into a sum of powers of linear forms and then we integrate each summand using the Maple procedure discussed above 6 Table 5 shows the minimum, average, and maximum computation times.

5.2. Integration of a monomial, using the iterated Laurent series. In this section, we test the implementation of the method of the iterated Laurent expansion described in Remark 15 of Section 3.47

Table 6] shows the results.

\footnotetext{
${ }^{6}$ This method is implemented in the Maple procedure integral_via_waring in waring.mpl.

${ }^{7}$ This method is implemented in the Maple procedure integral_via_iterated, defined in the file iterated_laurent.mpl.
} 
TABLE 6. Integration of a random monomial of prescribed degree using the iterated Laurent series

\begin{tabular}{|c|c|c|c|c|c|c|c|c|c|c|c|}
\hline \multirow[b]{2}{*}{$n$} & \multicolumn{11}{|c|}{ Degree } \\
\hline & 1 & 2 & 5 & 10 & 20 & 30 & 40 & 50 & 100 & 200 & 300 \\
\hline \multirow[t]{2}{*}{2} & $\begin{array}{l}0 \\
0\end{array}$ & $\begin{array}{l}0 \\
0 \\
0\end{array}$ & $\begin{array}{l}0 \\
\mathbf{0}\end{array}$ & $\begin{array}{l}0 \\
0\end{array}$ & $\begin{array}{l}0 \\
0\end{array}$ & $\begin{array}{l}0 \\
0\end{array}$ & $\begin{array}{l}0 \\
0.1\end{array}$ & $\begin{array}{l}0 \\
0.1\end{array}$ & $\begin{array}{l}0 \\
\mathbf{0} .5\end{array}$ & $\begin{array}{l}0.3 \\
\mathbf{3 . 0}\end{array}$ & $\begin{array}{l}0.2 \\
8.1\end{array}$ \\
\hline & 0 & 0.1 & 0.1 & 0.1 & 0.1 & 0.1 & 0.2 & 0.3 & 1.2 & 7.1 & 30 \\
\hline \multirow[t]{3}{*}{3} & 0 & 0 & 0 & 0 & 0 & 0 & 0.1 & 0.1 & 0.2 & & \\
\hline & 0 & 0 & 0 & 0.1 & 0.4 & 0.5 & 1.2 & 3.4 & 34 & & \\
\hline & 0 & 0 & 0.1 & 0.2 & 6.2 & 3.7 & 7.1 & 11 & 164 & & \\
\hline \multirow[t]{3}{*}{4} & 0 & 0 & 0 & 0 & 0.1 & 0.3 & 0.4 & 1.0 & & & \\
\hline & 0 & 0 & 0.1 & 0.3 & 1.4 & 4.8 & 16 & 39 & & & \\
\hline & 0 & 0.1 & 0.2 & 0.7 & 12 & 35 & 77 & 176 & & & \\
\hline \multirow[t]{3}{*}{5} & 0 & 0 & 0.1 & 0.1 & & 1.4 & 0.2 & & & & \\
\hline & 0.1 & 0.1 & 0.2 & 0.7 & 4.5 & 36 & 78 & & & & \\
\hline & 0.1 & 0.1 & & 12 & 35 & & & & & & \\
\hline \multirow[t]{3}{*}{6} & 0.1 & 0 & 0.1 & 0.2 & 0.4 & & & & & & \\
\hline & 0.1 & 0.2 & 0.3 & 1.6 & 24 & & & & & & \\
\hline & 0.1 & 5.0 & 0.6 & 5.9 & 205 & & & & & & \\
\hline \multirow[t]{3}{*}{7} & 0.1 & 0.1 & 0.2 & 0.4 & & & & & & & \\
\hline & 0.1 & 0.3 & 0.7 & 4.1 & & & & & & & \\
\hline & 0.2 & 5.4 & 4.6 & 22 & & & & & & & \\
\hline \multirow[t]{3}{*}{8} & 0.2 & 0.2 & 0.2 & 0.3 & & & & & & & \\
\hline & 0.2 & 0.3 & 1.0 & 11 & & & & & & & \\
\hline & 0.2 & 4.8 & 4.1 & 111 & & & & & & & \\
\hline \multirow[t]{3}{*}{10} & 0.3 & 0.4 & 0.4 & & & & & & & & \\
\hline & 0.5 & 0.5 & 2.8 & & & & & & & & \\
\hline & 7.0 & 3.5 & 12 & & & & & & & & \\
\hline \multirow[t]{3}{*}{15} & 1.3 & 1.4 & 3.5 & & & & & & & & \\
\hline & 1.7 & 1.9 & 25 & & & & & & & & \\
\hline & 5.8 & 5.1 & 73 & & & & & & & & \\
\hline \multirow[t]{3}{*}{20} & 3.8 & 4.0 & 4.7 & & & & & & & & \\
\hline & 5.0 & 5.3 & 123 & & & & & & & & \\
\hline & 9.4 & 8.1 & 352 & & & & & & & & \\
\hline \multirow[t]{2}{*}{30} & 25 & 26 & & & & & & & & & \\
\hline & $\begin{array}{r}29 \\
41\end{array}$ & $\begin{array}{l}\mathbf{2 9} \\
32\end{array}$ & & & & & & & & & \\
\hline \multirow{3}{*}{40} & & & & & & & & & & & \\
\hline & 98 & 101 & & & & & & & & & \\
\hline & 106 & 152 & & & & & & & & & \\
\hline \multirow[t]{3}{*}{50} & 248 & 259 & & & & & & & & & \\
\hline & 271 & 283 & & & & & & & & & \\
\hline & 300 & 429 & & & & & & & & & \\
\hline
\end{tabular}

5.3. Integration of a monomial, using Taylor expansion. Here, we test the implementation of the algorithm described in Section 3.4. This algorithm is based on 118

The running times are shown in Table 7.

5.4. Integration of a dense homogeneous polynomial over a simplex. Following the tests on single monomials, we ran tests on random polynomials of varying density. We generated these polynomials using the Maple function randpoly, requesting a number $r$ of monomials and the homogeneous degree $M$. For each monomial the exponent vector was drawn uniformly from $\left\{\mathbf{M} \in \mathbb{N}^{d}:|\mathbf{M}|=M\right\}$,

${ }^{8}$ This method is implemented in the Maple procedure integral_via_duality, defined in the file duality.mpl. 
TABLE 7. Integration of a random monomial of prescribed degree using Taylor expansion

\begin{tabular}{|c|c|c|c|c|c|c|c|c|c|c|c|}
\hline \multirow[b]{2}{*}{$n$} & \multicolumn{11}{|c|}{ Degree } \\
\hline & 1 & 2 & 5 & 10 & 20 & 30 & 40 & 50 & 100 & 200 & 300 \\
\hline \multirow[t]{2}{*}{2} & $\begin{array}{l}0 \\
\mathbf{0}\end{array}$ & $\begin{array}{l}0 \\
0\end{array}$ & $\begin{array}{l}0 \\
\mathbf{0}\end{array}$ & $\begin{array}{l}0 \\
0\end{array}$ & $\begin{array}{l}0 \\
0\end{array}$ & $\begin{array}{l}0 \\
0.1\end{array}$ & $\begin{array}{l}0 \\
\mathbf{0 . 2}\end{array}$ & $\begin{array}{l}0 \\
0.3\end{array}$ & $\begin{array}{l}0 \\
\mathbf{2} .2\end{array}$ & $\begin{array}{r}0.9 \\
\mathbf{2 4}\end{array}$ & $\begin{array}{r}1.9 \\
\mathbf{6 8}\end{array}$ \\
\hline & 0 & 0 & 0 & 0.1 & 0.1 & 0.3 & 0.6 & 0.9 & 13 & 101 & 426 \\
\hline \multirow[t]{3}{*}{3} & 0 & 0 & 0 & 0 & 0 & 0 & 0.1 & 0 & & & \\
\hline & 0 & 0 & 0 & 0.1 & 2.0 & 8.1 & 39 & 61 & & & \\
\hline & 0 & 0 & 0.1 & 0.4 & 12 & 60 & 277 & 512 & & & \\
\hline \multirow[t]{3}{*}{4} & 0 & 0 & 0 & 0 & & & & & & & \\
\hline & 0 & 0 & 0.1 & 1.5 & & & & & & & \\
\hline & 0 & 0 & 0.4 & 34 & & & & & & & \\
\hline \multirow[t]{3}{*}{5} & 0 & 0 & 0 & 0.1 & & & & & & & \\
\hline & 0 & 0 & 0.1 & 4.9 & & & & & & & \\
\hline & 0 & 0 & 0.6 & 48 & & & & & & & \\
\hline \multirow[t]{3}{*}{6} & 0 & 0 & 0 & 0.1 & & & & & & & \\
\hline & 0 & 0 & 0.4 & 29 & & & & & & & \\
\hline & 0 & 0 & 1.7 & 236 & & & & & & & \\
\hline \multirow[t]{3}{*}{7} & 0 & 0 & 0 & & & & & & & & \\
\hline & 0 & 0 & 0.5 & & & & & & & & \\
\hline & 0 & 0 & 1.7 & & & & & & & & \\
\hline \multirow[t]{3}{*}{8} & 0 & 0 & 0 & & & & & & & & \\
\hline & 0 & 0 & 1.1 & & & & & & & & \\
\hline & 0 & 0 & 16 & & & & & & & & \\
\hline \multirow[t]{2}{*}{10} & 0 & 0 & 0 & & & & & & & & \\
\hline & $\begin{array}{l}0 \\
0\end{array}$ & $\begin{array}{l}\mathbf{0} \\
0.1\end{array}$ & $\begin{array}{c}3.0 \\
33\end{array}$ & & & & & & & & \\
\hline \multirow[t]{3}{*}{15} & 0 & 0 & 0.1 & & & & & & & & \\
\hline & 0 & 0.1 & 20 & & & & & & & & \\
\hline & 0 & 0.1 & 64 & & & & & & & & \\
\hline \multirow[t]{3}{*}{20} & 0 & 0 & 1.2 & & & & & & & & \\
\hline & 0 & 0.7 & 81 & & & & & & & & \\
\hline & 0 & 28 & 205 & & & & & & & & \\
\hline \multirow[t]{2}{*}{30} & 0 & 0 & & & & & & & & & \\
\hline & $\begin{array}{l}\mathbf{0} \\
0.1\end{array}$ & & & & & & & & & & \\
\hline \multirow{3}{*}{40} & 0 & 0 & & & & & & & & & \\
\hline & 0.1 & 1.2 & & & & & & & & & \\
\hline & 0.1 & 23 & & & & & & & & & \\
\hline \multirow[t]{3}{*}{50} & 0.1 & 0.1 & & & & & & & & & \\
\hline & 0.1 & 1.7 & & & & & & & & & \\
\hline & 0.2 & 18 & & & & & & & & & \\
\hline
\end{tabular}

and the coefficient is drawn uniformly from $\{1, \ldots, 100\}$. Due to collisions, the generated polynomial can actually have fewer monomials than $r 9$

We only include the results for a family of randomly generated, very dense homogeneous polynomials, where we draw $r=\left(\begin{array}{c}M+d-1 \\ d-1\end{array}\right)$ random monomials. Table 10 shows the number of monomials in the resulting polynomials for our random tests. Tables 11, 13, 12 show the test results of the three methods.

We remark that in the case of the method using decompositions into powers of linear forms, we note that the same powers of linear forms appear in the decomposition formulas (13) for many different monomials $\mathbf{x}^{\mathbf{M}_{1}}, \mathbf{x}^{\mathbf{M}_{2}}$. We take advantage of this fact by collecting the coefficients of powers of linear forms 10

${ }^{9}$ This is implemented in the Maple procedure random_sparse_homogeneous_polynomial -with_degree in the file examples.mpl.

${ }^{10}$ This is implemented in the procedure list_integral_via_waring. 
TABLE 8. Integration of a monomial of prescribed degree with 2 effective variables by decomposition into a sum of powers of linear forms

\begin{tabular}{|c|c|c|c|c|c|c|c|c|c|c|c|}
\hline \multirow[b]{2}{*}{$n$} & \multicolumn{11}{|c|}{ Degree } \\
\hline & 1 & 2 & 5 & 10 & 20 & 30 & 40 & 50 & 100 & 200 & 300 \\
\hline \multirow[t]{3}{*}{3} & 0 & 0 & 0 & 0 & 0 & 0 & 0 & 0 & 1.9 & 1.9 & 9.8 \\
\hline & 0 & 0 & 0 & 0.1 & 0.3 & 0.7 & 1.5 & 2.2 & 11 & 47 & 109 \\
\hline & 0 & 0.2 & 0.1 & 0.2 & 0.5 & 1.1 & 2.3 & 3.5 & 15 & 80 & 217 \\
\hline \multirow[t]{3}{*}{4} & 0 & 0 & 0 & 0 & 0 & 0 & 0 & 0.5 & 0.1 & 0.2 & 0.3 \\
\hline & 0 & 0 & 0 & 0.1 & 0.4 & 0.9 & 1.7 & 2.8 & 13 & 64 & 180 \\
\hline & 0 & 0.3 & 0.2 & 0.2 & 0.7 & 1.4 & 2.8 & 4.3 & 20 & 103 & 270 \\
\hline \multirow[t]{3}{*}{5} & 0 & 0 & 0 & 0 & 0 & 0 & 0 & 0 & 1.8 & 0.3 & 6.0 \\
\hline & 0 & 0 & 0.1 & 0.1 & 0.6 & 1.3 & 2.5 & 3.5 & 17 & 81 & 205 \\
\hline & 0 & 0.4 & 0.2 & 0.3 & 1.0 & 2.0 & 3.8 & 5.9 & 26 & 128 & 335 \\
\hline \multirow[t]{3}{*}{6} & 0 & 0 & 0 & 0 & 0 & 0 & 0 & 0 & 2.1 & 7.0 & 0.3 \\
\hline & 0 & 0 & 0.1 & 0.2 & 0.7 & 1.8 & 3.1 & 4.5 & 25 & 105 & 241 \\
\hline & 0.2 & 0.2 & 0.2 & 0.4 & 1.2 & 2.6 & 4.9 & 7.5 & 34 & 164 & 449 \\
\hline \multirow[t]{3}{*}{7} & 0 & 0 & 0 & 0 & 0 & 0 & 0 & 0 & 2.3 & 0.2 & 0.2 \\
\hline & 0 & 0 & 0.1 & 0.2 & 1.0 & 1.9 & 3.7 & 5.9 & 24 & 128 & 279 \\
\hline & 0 & 0.3 & 0.3 & 0.5 & 1.5 & 3.0 & 5.9 & 8.7 & 39 & 184 & 468 \\
\hline \multirow[t]{3}{*}{8} & 0 & 0 & 0 & 0 & 0 & 0 & 0 & 0.1 & 4.2 & 0.4 & 10.0 \\
\hline & 0 & 0 & 0.1 & 0.3 & 1.1 & 2.3 & 4.0 & 6.8 & 28 & 122 & 347 \\
\hline & 0 & 0.5 & 0.3 & 0.6 & 1.6 & 3.5 & 6.5 & 10.0 & 45 & 206 & 529 \\
\hline \multirow[t]{3}{*}{10} & 0 & 0 & 0 & 0 & 0 & 0 & 0 & 1.7 & 0.1 & 13 & \\
\hline & 0 & 0 & 0.1 & 0.4 & 1.3 & 3.3 & $\mathbf{5} .6$ & 10 & 41 & 189 & \\
\hline & 0.3 & 0.3 & 0.4 & 0.7 & 2.2 & 4.9 & 8.6 & 14 & 58 & 266 & \\
\hline \multirow[t]{3}{*}{15} & 0 & 0 & 0 & 0 & 0.1 & 0.1 & 0.1 & 0.1 & 0.2 & 0.2 & \\
\hline & 0.1 & 0.1 & 0.2 & 0.7 & 2.4 & 6.0 & 9.9 & 15 & 65 & 292 & \\
\hline & 0.3 & 0.3 & 0.5 & 1.2 & 4.0 & 8.9 & 16 & 24 & 105 & 479 & \\
\hline \multirow[t]{3}{*}{20} & 0 & 0.1 & 0 & 0.1 & 0.1 & 0.1 & 0.1 & 0.2 & 23 & & \\
\hline & 0.1 & 0.2 & 0.5 & 1.3 & 4.7 & 9.7 & 17 & 26 & 123 & & \\
\hline & 0.3 & 0.5 & 0.8 & 2.1 & 7.1 & 15 & 26 & 40 & 170 & & \\
\hline \multirow[t]{3}{*}{30} & 0.1 & 0.2 & 0.2 & 0.2 & 0.2 & 0.2 & 0.2 & 0.2 & 0.3 & & \\
\hline & 0.2 & 0.4 & 0.9 & 2.8 & 11 & 19 & 38 & 55 & 254 & & \\
\hline & 0.4 & 0.8 & 1.5 & 4.7 & 15 & 33 & 58 & 92 & 369 & & \\
\hline \multirow[t]{3}{*}{40} & 0.3 & 0.3 & 0.3 & 0.3 & 0.3 & 0.4 & 0.4 & 0.4 & & & \\
\hline & 0.4 & 0.6 & 1.9 & 5.4 & 20 & 41 & 66 & 101 & & & \\
\hline & 0.6 & 1.2 & 2.9 & 8.5 & 29 & 62 & 110 & 171 & & & \\
\hline \multirow[t]{3}{*}{50} & 0.5 & 0.5 & 0.5 & 0.6 & 0.6 & 0.6 & 0.7 & 36 & & & \\
\hline & 0.6 & 1.0 & 3.4 & 9.2 & 31 & 63 & 130 & 201 & & & \\
\hline & 0.8 & 2.1 & 4.7 & 14 & 49 & 106 & 185 & 286 & & & \\
\hline
\end{tabular}

5.5. Integration of a monomial with few effective variables. Finally, we tested the performance of the three algorithms on monomials $\mathrm{x}^{\mathrm{M}}$ with a small number $D$ of effective variables. We fix the number $D$. Then, for a given dimension $n \geq D$ and total degree $M$, we picked 50 combinations of a random simplex $\Delta$ of dimension $n$ and a random exponent vector $\mathbf{M}=\left(M_{1}, \ldots, M_{D}, 0, \ldots, 0\right)$ with $|\mathbf{M}|=M$. We only include the results for $D=2$ in Tables 8 , and 14 .

5.6. Discussion. In our implementation of the three methods and our experiments for the case of random monomials, we observe that the method of iterated Laurent expansion is faster than the two other methods if the dimension $n$ is very small (up to $n=5$ ). Starting from dimension $n=6$, the method using decompositions into powers of linear forms is faster than the other two methods. The method using Taylor expansion is always inferior to the better of the two other methods, for any combination of degree and dimension.

In the experiments with random dense polynomials, in our implementation we did not see significant savings from collecting the coefficients of the same powers of 
linear forms. As a consequence, the ranking of the three methods is the same as it is in the case of random monomials.

The experiments with random monomials with few effective variables show that all three methods benefit from using few effective variables. The greatest effect is on the method using decompositions into powers of linear forms, where, for example, the restriction to 2 effective variables allows us to handle combinations of high degree $M=200$ and high dimension $n=15$. However, for low dimensions $(n \leq 5)$, the method of iterated Laurent expansion still wins. Also, here the method using Taylor expansion is always inferior to the better of the two other methods. This discussion shows the power of Brion's formula.

5.7. Connections to numerical integration. Although all of our calculations above involved exact rational arithmetic, it is a natural question to ask what are the implications of our method for numerical computation. Of course, one obvious consequence is that our algorithms can be used to calibrate floating point calculations.

On the suggestion of a referee we also tested the performance of our algorithms in a floating point setting. We modified the Maple programs to use IEEE 754 doubleprecision floating point numbers instead of using exact rational arithmetic, using Maple's hfloat functions. We did not make any attempt to change the algorithms to reduce floating point errors.

We also modified our examples so that all function evaluations and the final results could fit into the floating point range. To this end, we chose the coordinates of our simplices to be floating point numbers between 0.95 and 1.05. For simplicity we restricted the tests to the integration of monomial functions in the same combinations of degree and dimension as in the experiments with exact arithmetic. For each combination, we tested 50 examples in each algorithm. To our surprise the numerical calculations were quite interesting (we do not include the five pages of tables here, but they are available from the authors upon request). Here is a summary:

- iterated produces meaningful results (with maximum relative errors $\leq$ $10 \%$ ) for $n=1, M \leq 4$; for $n=2, M \leq 5$; for $n=3, M \leq 1$; and for $n=4$, $M \leq 1$. For higher combinations of degree and dimension, the results are off by orders of magnitude.

- waring works well for $n=1$ for all tested degrees up to $M=1000$; for $n=2, M \leq 10$; for $n=3, M \leq 5$; and for $n=4, M=1$.

- duality works well for $n=1, M \leq 100$; for $n=2, M \leq 30$; for $n=3$, $M \leq 20$; for $n=4, M \leq 10$; and generally for all low degrees $M \leq 5$ that could be computed within the time limit, i.e., for $n \leq 12$ and $M \leq 5$; and for $n \leq 50$ and $M \leq 2$.

We then decided to run these kinds of integrals using CUBPACK [16, a stateof-the-art Fortran 90 package for numerical integration using cubature formulas. CUBPACK contains an implementation of an adaptive algorithm for the integration over simplices of arbitrary dimension by Genz and Cools [22], on the basis of the famous Grundmann-Möller cubature rule [23]. This code allows us to integrate arbitrary functions given by point evaluations. A subdivision method is used to improve error estimates. We ran the CUBPACK experiments on a Sun Fire V440 
with UltraSPARC-IIIi processors running at $1.6 \mathrm{GHz}$, using the Sun Fortran 95 compiler, version 8.2.

In our experiments, we asked CUBPACK to compute the integral to a relative error of $5.0 \times 10^{-4}$ and allowed a maximum of 200 million function evaluations, a limit that was by far never reached in any of the tests 11 The results of the experiments are as follows. All computations using CUBPACK finished in a fraction of a second. CUBPACK returned results for the tests in all dimensions up to 33 (and all degrees up to 1000), but it failed with floating point exceptions if the dimension was at least 34 .

For the combinations of degree and dimension where our Maple programs finished within 600 seconds, we then compared the exact results computed by our programs to the numerical results of CUBPACK. Table 16 shows the average relative errors. In low dimensions (up to 12), the actual errors are always well below the error estimation provided by CUBPACK, for dimensions up to 4 by about 2 orders of magnitude. However, starting from dimension 14, even for low degree, the numerical results using CUBPACK are off by several orders of magnitude, which is not indicated by the provided error estimate. The reason for the failure of the CUBPACK error estimators in this situation is unknown to us.

\section{Conclusions}

We have discussed various algorithms for the exact integration of polynomials over simplicial regions. Besides their theoretical efficiency, the simple rough experiments we performed clearly demonstrated that these methods are robust enough to attack rather difficult problems. Our investigations opened several doors for further development, which we will present in a forthcoming paper.

First, we have some theoretical issues expanding from our results. As in the case of volumes and the computation of centroids, it is likely that our hardness result, Theorem 1, can be extended into an inapproximability result as those obtained in 34. Another goal is to study other families of polytopes for which exact integration can be done efficiently. Furthermore, we will present a natural extension of the computation of integrals, the efficient computation of the highest degree coefficients of a weighted Ehrhart quasipolynomial of a simplex. Besides the methods of the present article, these last computations are based on the results of [7] and [6].

Second, our intention has been all along to develop algorithms with a good chance of becoming practical and that allow for clear implementation. Thus we also have some practical improvements to discuss. For example, in order to develop practical integration software, it appears that our methods should be coupled with fast techniques for decomposing domains into polyhedral regions (e.g. triangulations).

Finally, our experiments indicated that our exact integration algorithms are not only useful to calibrate any numerical technique, but they may even be useful already within the context of floating point computation. In particular, the method 'duality' shows good promise for floating point computations for problems in high dimension and low degree. Obviously, a serious floating point implementation and error analysis would be necessary, but this is beyond the scope of this paper.

\footnotetext{
${ }^{11}$ The Fortran driver for CUBPACK is contained in the file pisa-test.f90; the Maple proce-
} dure that calls it is contained in the file cubpack.mpl. 
Note. Appendix A, which contains additional computational tables, 9-16, will appear as a link in an electronic supplement to this article.

Note added in proof: Since the acceptance of this paper, our students Stanislav Moreinis and Jianqiu $\mathrm{Wu}$ have created a new implementation of the method of integration by decomposition into powers of linear forms. The implementation is in $\mathrm{C}++$ and has yielded speedups of several orders of magnitude. The new implementation and a detailed report on it will be made available.

\section{ACKNOWLEDGEMEnTS}

Part of the work for this paper was done while the authors visited Centro di Ricerca Matematica Ennio De Giorgi at the Scuola Normale Superiore of Pisa, Italy. We are grateful for the hospitality of CRM and the support we received. The third author was also supported by NSF grant DMS-0608785. We are grateful for help from Christophe Margerin and comments from Bruce Reznick, Jean-Bernard Lasserre, and Bernd Sturmfels. Last but not least, we thank the two anonymous referees who gave us useful comments and corrections.

\section{REFERENCES}

[1] J. Alexander and A. Hirschowitz, Polynomial interpolation in several variables, J. Algebraic Geom. 4 (1995), 201-222. MR1311347 (96f:14065)

[2] V. Baldoni, N. Berline, J. A. De Loera, M. Köppe, and M. Vergne, Maple programs accompanying the manuscript How to integrate a polynomial over a simplex, http://www.math.ucdavis.edu/ mkoeppe/art/pisa-integration-experiments/, 2008.

[3] A. I. Barvinok, Computation of exponential integrals, Zap. Nauchn. Sem. Leningrad. Otdel. Mat. Inst. Steklov. (LOMI) Teor. Slozhn. Vychisl. 5 (1991), 149-162, 175-176, translation in J. Math. Sci. 70 (1994), no. 4, 1934-1943. MR1118837 (92i:65228)

[4] _ Exponential integrals and sums over convex polyhedra (in Russian), Funktsional. Anal. i Prilozhen. 26 (1992), no. 2, 64-66, translated in Funct. Anal. Appl. 26 (1992), no. 2, pp. 127-129. MR.1173086 (93g:32052)

[5] , Partition functions in optimization and computational problems, Algebra i Analiz 4 (1992), 3-53, translation in St. Petersburg Math. J. 4 (1993), no. 1, pp. 1-49. MR1171953 (93f:90131)

[6] N. Berline and M. Vergne, Local Euler-Maclaurin formula for polytopes, Moscow Math. J. 7 (2007), 355-386. MR2343137 (2008k:52026)

[7] L Local Euler-Maclaurin expansion of Barvinok valuations and Ehrhart coefficients of rational polytopes, Contemporary Mathematics 452 (2008), 15-33. MR 2343137 (2008k:52026)

[8] N. Berline, E. Getzler, and M. Vergne, Heat kernels and Dirac operators, Grundlehren der Mathematischen Wissenschaften [Fundamental Principles of Mathematical Sciences], vol. 298, Springer-Verlag, Berlin, 1992. MR,1215720 (94e:58130)

[9] A. T. Bharucha-Reid and M. Sambandham, Random polynomials, Academic Press, Orlando, Florida, 1986. MR 856019 (87m:60118)

[10] J. Brachat, P. Comon, B. Mourrain, and E. Tsigaridas, Symmetric tensor decomposition, arXiv: 0901.3706v2 [cs.SC], 2009.

[11] M. C. Brambilla and G. Ottaviani, On the Alexander-Hirschowitz theorem, e-print arXiv:math.AG/0701409v2, 2007.

[12] G. Brightwell and P. Winkler, Counting linear extensions, Order 8 (1991), no. 3, 225-242. MR:1154926 (93b:06002)

[13] M. Brion, Points entiers dans les polyédres convexes, Ann. Sci. École Norm. Sup. 21 (1988), no. 4, 653-663. MR982338 (90d:52020)

[14] M. Brion and M. Vergne, Lattice points in simple polytopes, J. Amer. Math. Soc. 10 (1997), no. 2, 371-392. MR.1415319 (98a:11132) 
[15] P. Bürgisser, M. Clausen, and M. A. Shokrollahi, Algebraic complexity theory, Grundlehren der mathematischen Wissenschaften, no. 315, Springer, Berlin, 1997. MR.1440179 (99c:68002)

[16] R. Cools and A. Haegemans, Algorithm 824: CUBPACK: a package for automatic cubature; framework description., ACM Trans. Math. Software 29 (2003), no. 3, 287-296.

[17] W. Dahmen, On multivariate B-splines, SIAM J. Numer. Anal. 17 (1980), no. 2, 179-191. MR567267 (81c:41020)

[18] J. A. De Loera, J. Rambau, and F. Santos, Triangulations: Structures for algorithms and applications, Algorithms and Computation in Mathematics, vol. 25, Springer-Verlag, Berlin, 2010.

[19] M. E. Dyer and A. M. Frieze, On the complexity of computing the volume of a polyhedron, SIAM J. Comput. 17 (1988), no. 5, 967-974. MR961051 (90f:68077)

[20] G. Elekes, A geometric inequality and the complexity of computing volume, Discrete Comput. Geom. 1 (1986), no. 4, 289-292. MR866364 (87k:68138)

[21] M. R. Garey and D. S. Johnson, Computers and intractibility: A guide to the theory of NP-completeness, W. H. Freeman and Co., San Francisco, California, 1979. MR519066 (80g:68056)

[22] A. Genz and R. Cools, An adaptive numerical cubature algorithm for simplices, ACM Trans. Math. Software 29 (2003), no. 3, 297-308. MR2002733 (2004g:65021)

[23] A. Grundmann and H. M. Möller, Invariant integration formulas for the n-simplex by combinatorial methods, SIAM J. Numer. Anal. 15 (1978), 282-290. MR488881 (81e:41045)

[24] R. Kannan and A. Bachem, Polynomial algorithms for computing the Smith and Hermite normal forms of an integer matrix, SIAM J. Comput. 8 (1979), no. 4, 499-507. MR573842 (81k:15002)

[25] L. Khachiyan, Complexity of polytope volume computation, New trends in discrete and computational geometry, Algorithms Combin., vol. 10, Springer, Berlin, 1993, pp. 91-101. MR.1228040 (94m:68190)

[26] J. B. Lasserre, Integration on a convex polytope, Proceedings of the AMS 126 (1998), no. 8, 2433-2441. MR1459132 (98j:65016)

[27] J. B. Lasserre and K. Avrachenkov, The multidimensional version of $\int_{a}^{b} x^{p} \mathrm{~d} x$, American Mathematical Monthly 108 (2001), no. 2, 151-154. MR.1818188 (2002g:26015)

[28] M. Laurent, Sums of squares, moment matrices, and optimization over polynomials, In "Emerging applications of algebraic geometry" (Minneapolis, Minnesota) (M. Putinar and S. Sullivant, eds.), IMA volumes in Mathematics and its Applications, 2008. MR2500468

[29] J. Lawrence, Polytope volume computation, Math. Comp. 57 (1991), no. 195, 259-271. MR 1079024 (91j:52019)

[30] S. Lin, B. Sturmfels, and Z. Xu, Marginal likelihood integrals for mixtures of independence models, J. Mach. Learn. Res. 10 (2009), 1611-1631. MR2534873

[31] G. Matera, Integration of multivariate rational functions given by straight-line programs, Proceedings of the 11th international symposium on applied algebra, algebraic algorithms and error-correcting codes (London), Lecture Notes in Computer Science, vol. 948, Springer, 1995, pp. 347-364. MR:1448176 (98c:68104)

[32] C. A. Micchelli, Mathematical aspects of geometric modeling, CBMS-NSF Regional Conference Series in Applied Mathematics, vol. 65, Society for Industrial and Applied Mathematics (SIAM), Philadelphia, PA, 1995. MR 1308048 (95i:65036)

[33] T. S. Motzkin and E. G. Straus, Maxima for graphs and a new proof of a theorem of Turán, Canadian Journal of Mathematics 17 (1965), 533-540. MR0175813 (31:89)

[34] L. Rademacher, Approximating the centroid is hard, Proceedings of 23th annual ACM Symposium of Computational Geometry, Gyeongju, South Korea, June 6-8, 2007, 2007, pp. 302-305. MR.2469178 (2009m:68104)

[35] M. Schechter, Integration over a polyhedron: An application of the Fourier-Motzkin elimination method, American Mathematical Monthly 105 (1998), no. 3, 246-251. MR.1615560 (99d:15014)

[36] Z. Xu, Multivariate splines and polytopes, arXiv: 0806.1127v3, 2009.

[37] G. M. Ziegler, Lectures on polytopes, Graduate texts in Mathematics, no. 152, Springer, New York, 1995. MR1311028 (96a:52011)

[38] O. C. Zienkiewicz and R. L. Taylor, The finite element method, McGraw-Hill, London, 1988. 
Dipartimento di Matematica, Università degli studi di Roma "Tor Vergata", Via DELLA RICERCA SCIENTIFICA 1, I-00133, ITALY

E-mail address: baldoni@mat.uniroma2.it

Centre de Mathématiques Laurent Schwartz, École Polytechnique, 91128 Palaiseau Cedex, France

E-mail address: nicole.berline@math.polytechnique.fr

Department of Mathematics, University of California, Davis, One Shields Avenue, Davis, CALifornia 95616

E-mail address: deloera@math.ucdavis.edu

Department of Mathematics, University of California, Davis, One Shields Avenue, Davis, CALifornia 95616

E-mail address: mkoeppe@math.ucdavis.edu

Institut de Mathématiques de Jussieu, 175 rue du Chevaleret, 75103 Paris, France

E-mail address: vergne@math.jussieu.fr 